September 3, 2021

\title{
Collagen polarization promotes epithelial elongation by stimulating locoregional cell proliferation.
}

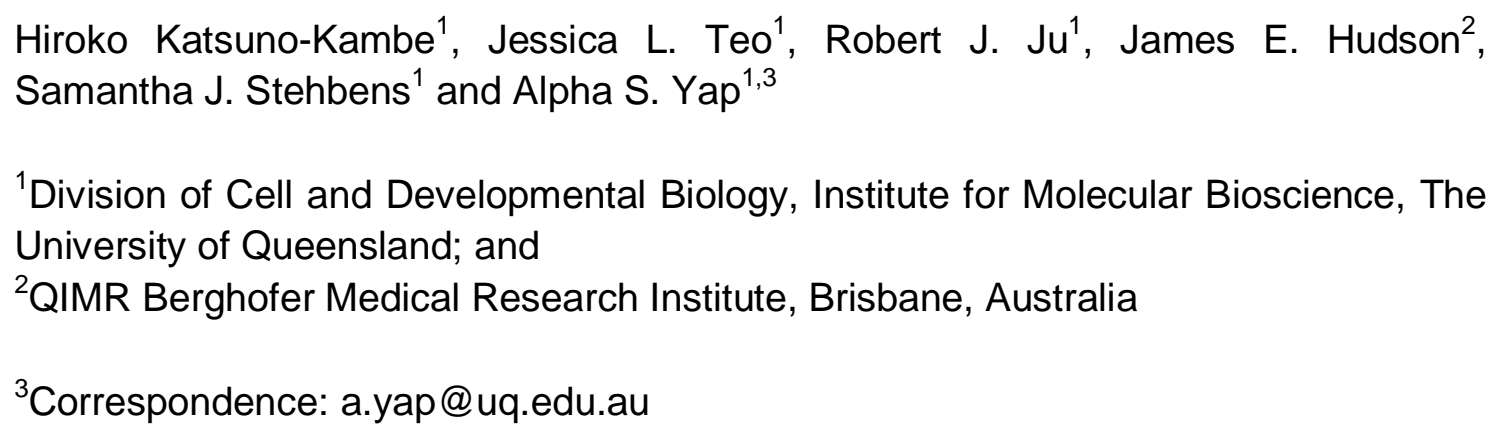

Keywords: Epithelia, elongation, tubulogenesis, collagen I, symmetry breaking, anisotropy

\section{Abstract}

Epithelial networks are commonly generated by processes where multicellular aggregates elongate and branch. Here we focus on understanding cellular mechanisms for elongation, using an organotypic culture system as a model of mammary epithelial anlage. Isotropic cell aggregates broke symmetry and slowly elongated when transplanted into collagen 1 gels. The elongating regions of aggregates displayed enhanced cell proliferation that was necessary for elongation to occur. Strikingly, this loco-regional increase in cell proliferation occurred where collagen 1 fibrils reorganized into bundles which were polarized with the elongating aggregates. Applying external stretch as a cell-independent way to reorganize the ECM, we found that collagen polarization stimulated regional cell proliferation to precipitate symmetry-breaking and elongation. This required $\beta 1$-integrin and ERK signaling. We propose that collagen polarization supports epithelial anlagen elongation by stimulating loco-regional cell proliferation. This could provide a longlasting structural memory of the initial axis that is generated when anlage break symmetry.

\section{Introduction}

Branched tubules represent one of the archetypal modes of epithelial organization (Iruela-Arispe and Beitel, 2013; Varner and Nelson, 2014). In organs such as the mammary gland and lungs, networks of hollow epithelial tubes mediate the physiological exchange of gases, nutrients and solutes between the body and its external environment (Chung and Andrew, 2008). These definitive networks are established by a complex morphogenetic process, where tubules grow outwards 
45 from their precursors until they are instructed to branch, after which outgrowth 46 continues until the network is completed (Affolter et al., 2009; Andrew and Ewald, 47 2010). The branching and elongation of multicellular aggregates can thus be considered as fundamental processes in the generation of tubular networks.

Interestingly, different organs also use distinct strategies for tubulogenesis. For example, in the trachea, lumens appear early and accompany the growth of tubules (Schottenfeld et al., 2010), whereas in the salivary and mammary glands tubules first begin as non-polarized cellular aggregates (or anlage), which then elongate as solid, multicellular cords before eventually forming lumens (BastidasPonce et al., 2017; Nerger and Nelson, 2018; Tucker, 2007). In the present study we use the mammary epithelium as a model to analyse what guides the elongation of multicellular anlage.

Tubulogenesis is a highly regulated phenomenon. The decision to branch is recognized to be a critical checkpoint that is controlled by developmental signals and cell-cell and cell-ECM interactions (Goodwin and Nelson, 2020). The elongation of tubule precursors is also thought to be a regulated process controlled by receptor tyrosine kinases and other signaling pathways (Costantini and Kopan, 2010; Gjorevski and Nelson, 2010; Sternlicht et al., 2006). However, elongation occurs over hours to days, time scales that are much longer than the underlying cellular processes and the signaling pathways that guide them. This raises the question of whether there may be mechanisms that can help guide elongation over longer time scales, effectively serving as a bridge between the rapidity of cell signaling and the slow progression of macroscopic anlage elongation. In this study, we show how collagen 1 within the extracellular matrix (ECM) can provide such a bridge by stimulating cell proliferation.

The ECM comprises complex mixtures of proteins, glycosaminoglycans and glycoconjugates that fill the extracellular spaces of tissues and organs (Frantz et al., 2010). One of the main components of ECM is collagen, particularly type 1 collagen (also called collagen 1), which is often the dominant form during epithelial tubulogenesis (Graham et al., 1988; Keely et al., 1995; Llacua et al., 2018; Nakanishi et al., 1986; Simon-Assmann et al., 1995). Type 1 collagen exerts a diverse range of effects that can potentially influence epithelial elongation. Fibrillar collagen helps scaffold other molecules, such other ECM proteins and growth factors (Kanematsu et al., 2004; Wipff and Hinz, 2008). Adhesion between cells and ECM allows the chemical and mechanical properties of the ECM to regulate cell signaling and gene regulation (Shi et al., 2011). These cell-ECM adhesions also allow cell-based forces to reorganize the ECM. In particular, cells can rearrange collagen fibrils to influence cell migration (Buchmann et al., 2021; Gjorevski et al., 2015; Guo et al., 2012; Shi et al., 2014).

Importantly, fibrillar components of the ECM, such as collagen 1, are relatively long-lived (Price and Spiro, 1977; Verzijl et al., 2000), making them attractive 86 candidates to bridge time scales during the elongation process. Indeed, rearrangement of collagen has been implicated in patterning epithelial branching 88 (Brownfield et al., 2013; Guo et al., 2012; Harunaga et al., 2011; Ingman et al., 2006; 
89 Patel et al., 2006). However, it is difficult to elucidate the specific contribution for 90 collagen in the complex environment of an organ, where there are many additional 91 contributions from other cell types and various chemotactic signals. Therefore, in this study we used three-dimensional (3D) organotypic cultures to test how the extracellular matrix $(\mathrm{ECM})$ regulates epithelial elongation. We report that a collagen 1 matrix induces the elongation of mammary epithelial anlage. This is accompanied by the polarization of the matrix itself, an event that sustains anlagen elongation by stimulating cell proliferation.

\section{Results}

\section{An experimental system to capture symmetry-breaking and elongation of epithelial aggregates.}

To model the elongation of epithelial anlage, we induced multicellular aggregates to break symmetry by manipulating the ECM environment in which the cells were grown. When MCF10A cells are grown on Matrigel ${ }^{\mathrm{TM}}$ substrates and in media supplemented with soluble Matrigel ${ }^{\mathrm{TM}}$ (Debnath et al., 2003), they proliferate from single isolated cells to form a multicellular aggregate, which then polarizes and clears the central mass of apoptotic cells, generating a central lumen in a process known as cavitation (Figure 1-figure supplement 1A). In contrast, when isolated cells were embedded in a gel of Type 1 collagen, which is the major ECM component in the stromal environment during mammary tubulogenesis (Schedin and Keely, 2011), they proliferated to form elongated, solid cords of non-polarized cells (Figure 1-figure supplement 1B) (Krause et al., 2008). This indicated that some properties of the collagen 1 environment might provide an instructive cue for elongation.

In order to capture the process by which isotropic aggregates broke symmetry and then elongated, cells were first seeded in Matrigel $^{\mathrm{TM}}$ until proliferation arrested at 10 days (Figure 1A); aggregates were then isolated and embedded into collagen 1 (acid solubilized Rat Type I collagen, 37$\square$ for 30 minutes). Lumens were apparent in some Matrigel-embedded aggregates, but most remained as solid spheres, resembling anlage. Live-cell imaging revealed that the transplanted aggregates displayed small jiggling motions for several hours, then spontaneously broke symmetry and elongated to form cord-like structures similar to those formed when cultured in collagen from the outset (Figure 1B and Video 1). These elongating aggregates were generally solid, with no evident lumens (Figure 2A). Commonly, elongation began with a group of cells that protruded away from the more spherical original aggregate (Figure 1C,E). In contrast, when aggregates were transplanted from Matrigel ${ }^{\mathrm{TM}}$ back into Matrigel $^{\mathrm{TM}}$, they grew slightly but did not elongate (Figure 1-figure supplement 1C). Therefore, transplantation into a collagen I matrix effectively caused isotropic MCF10A aggregates to break symmetry and elongate. 
133 Multiple cellular processes have been implicated in epithelial elongation (Andrew and

134 Ewald, 2010; Economou et al., 2013; Keller, 2002). We first examined cell 135 proliferation, given the evident increase in the size of aggregates that elongated. 136 Staining for Ki-67, a nonhistone nuclear protein commonly used as a marker of 137 proliferating cells (Soliman and Yussif, 2016), showed clearly evident proliferation in 138 aggregates that had elongated $8 \mathrm{~d}$ after transplantation into collagen (Figure 1C,D). 139 These elongating aggregates commonly consisted of elongated extensions as well 140 as a rounded region that marked the original aggregate (Figure 1B). Strikingly, Ki-67141 positive cells were more frequent in the elongating parts of the aggregates (41.00 \pm $1424.55 \%$ of all cells) compared with the non-elongating parts of the aggregates (18.86 $143 \pm 3.05 \%$ ) (Figure 1C,D). Live-cell imaging of cells expressing NLS-mCherry also 144 showed that the proportion of nuclei which divided was higher in the elongating than 145 the non-elongating areas within aggregates (Figure 1E,F). Thus, elongation was 146 associated with loco-regional differences in cell proliferation, which was enhanced in those parts of the aggregates that elongated.

More detailed inspection revealed that increased proliferation was first evident in aggregates two days after transplantation (Figure 1G) and appeared to precede the onset of symmetry-breaking (Figure $1 \mathrm{H}$ ). To quantitate aggregate elongation we calculated a symmetry ratio, i.e., the ratio of the maximum length $\left(L_{1}\right)$ and width $\left(L_{2}\right)$ of the aggregates. Completely round aggregates will have a symmetry ratio of 1 , whereas for elongated aggregates $L_{1} / L_{2}$ will be $>1$ (Figure 2B). The symmetry ratio first increased at Day 3 (Figure $1 \mathrm{H}$ ), approximately $24 \mathrm{~h}$ after an increase in proliferation was detected (Figure $1 \mathrm{G}$ ). To test if any loco-regional differences in proliferation were to be found at these early stages of symmetry breaking, we analysed aggregates that had broken symmetry (defined experimentally as a symmetry ratio $>1.5$ ) after 3 days of culture. We compared the proportion of cells that were Ki67-positive in the elongating regions of aggregates with the proportion to be found in the non-elongating areas of the same aggregates (Figure 1I). Ki67 positivity was two-fold greater in the elongating areas (Figure 1I), suggesting that loco-regional differences in proliferation were established early in the elongation process.

Importantly, elongation was inhibited when proliferation was blocked using the cell cycle inhibitors mitomycin $\mathrm{C}$ and aphidicolin (Figure $1 \mathrm{~J}$ ). In control cultures the proportion of Ki-67-positive cells increased progressively after transplantation into collagen (from $2.28 \pm 0.85 \%$ at Day 1 to $26.63 \pm 3.34 \%$ at Day 8 ). However, cell proliferation was significantly reduced by mitomycin C (3.88 $\pm 1.18 \%$, Day 8$)$ and aphidicolin (11.54 $\pm 3.55 \%$, Day 8) (Figure 1K). Aggregate elongation was also significantly reduced by both mitotic inhibitors (Figure $1 \mathrm{~J}$ ). Whereas the symmetry ratio increased as control anlage elongated, this was blocked by both mitomycin $\mathrm{C}$ and aphidicolin (Figure 1L). Formally, cell proliferation might have been necessary to initiate symmetry-breaking and cell elongation or ongoing proliferation might also have served to sustain elongation. To pursue this, we added mitomycin $C$ or aphidicolin after the first signs of symmetry breaking were evident (at 3 days after 176 transplantation). This reduced elongation by 3-fold (Figure 1M). Therefore, cell 
177 proliferation was required for elongation, with the capacity to contribute early in the

178 symmetry-breaking process and also later to sustain elongation.

179 We then used live cell imaging of labelled nuclei to evaluate other processes 180 implicated in epithelial elongation. Cells within the elongating regions tended to 181 divide along the axis of elongation (Figure 1-figure supplement 2A), as revealed by 182 comparing the axis of cell division and the principal axis of the region. The angle 183 difference between these axes was smaller in elongating areas compared with non184 elongating areas within the same aggregate (Figure 1-figure supplement 2A). This 185 suggested that polarized cell division accompanied enhanced proliferation during 186 aggregate elongation (Gong et al., 2004; Keller, 2006).

$187 \quad$ Tracking also revealed that cells were motile within elongating aggregates 188 (Figure 1-figure supplement 2B). This was evident in rounded aggregates that had 189 not broken symmetry and as well as in aggregates that had elongated (Figure 1190 figure supplement 2C). When we examined regional differences within aggregates 191 that had undergone elongation, we found that the speeds of migration were identical 192 in the parts that were elongating, compared with the non-elongating regions of the 193 aggregates (Figure 1-figure supplement 2D). However, the straightness of the tracks, 194 used an index of the persistence of migration, was slightly greater in the elongating 195 areas than in the non-elongating areas (Figure 1-figure supplement 2E). Furthermore, 196 cells within regions of elongation appeared to orient better with the axis of the 197 aggregate than did cells found in non-elongating areas. We measured this by 198 comparing the orientation of the tracks with the principal axis of the aggregates 199 (track displacement angle, Figure 1-figure supplement 2F). The track displacement

213 To gain further insight into the process of elongation, we sought to identify changes 214 in the ECM during this process. Consistent with the change in matrix environment angle was less in the elongating areas than in the non-elongating areas.

Inhibition of proliferation with either mitomycin $\mathrm{C}$ or aphidicolin did not affect cell migration speeds, in either the rounded or elongated areas (Figure 1-figure supplement 2C). However, blocking proliferation slightly reduced track straightness, implying a reduction in persistence (Figure 1-figure supplement 2G) and increased the track displacement angle (Figure 1-figure supplement $2 \mathrm{H}$ ). Therefore, the apparently orderly migration of cells was compromised by blocking cell proliferation. Together, these results suggest that proliferation was essential for the elongation process and may also have influenced aspects of directional migration that would be predicted to reinforce the elongation process.

\section{Collagen I condenses around epithelial aggregates that break symmetry.} associated with the transplantation process, re-embedded aggregates lost laminin $\mathrm{V}$ expression over time (Figure 2-figure supplement 1A). They expressed fibronectin throughout the experiments without any evident regional differences in the elongating areas of aggregates (Figure 2-figure supplement 1B).

Then we visualized Collagen 1 by labelling with the collagen-binding peptide CNA35 (mCherry-CNA35; (Krahn et al., 2006)), mixed with soluble Collagen I before 
221 the incorporation of MCF10A aggregates (Figure 2A). Collagen I, in contrast to the 222 basement membrane-like Matrigel $^{\mathrm{TM}}$, is composed of fibrillar polymers that can guide 223 cell movement and whose organization is influenced by the application of cellular 224 forces (Brownfield et al., 2013; Gjorevski et al., 2015; Piotrowski-Daspit et al., 2017). 225 Aggregate elongation appeared to coincide with change in fibril organization. 226 Whereas fibrils appeared isotropic in acellular gels, collagen condensed around the 227 aggregates that had begun to elongate, forming dense bands that extended away 228 from the cells into the gel. However, it was seldom possible to visualize individual 229 fibrils with the low magnification, long working distance lenses that were required to 230 visualize aggregates within the gels.

231 Therefore, we measured the coherency (or co-orientation) of gels with 232 Orientation J, which calculates the local orientation and isotropy for each pixel in an 233 image based on the structure tensor for that pixel (see Methods for 234 details;(Rezakhaniha et al., 2012)). We interpret coherency as reflecting collagen 235 bundling and condensation (which we shall call "bundling" for short), such as has 236 been observed elsewhere during elongation (Brownfield et al., 2013; Buchmann et 237 al., 2021; Gjorevski et al., 2015). Then, we compared collagen coherency with 238 aggregate shape, as measured by the symmetry ratio (Figure 2B), after 8 days 239 culture, when elongation was established. Overall, collagen coherency increased 240 with the increase in symmetry ratio (Figure $2 \mathrm{C}$ ), implying that the degree of collagen 241 bundling increased with aggregate elongation. Furthermore, closer examination 242 around elongating aggregates showed that collagen coherency was greater in 243 regions proximate to the elongating parts of the aggregates compared with the non244 elongating parts (Figure 2D). In contrast, aggregates that remained spherical 245 showed no regional differences in collagen organization. Similarly, earlier studies 246 showed that collagen reorganized ahead of the tips of elongating aggregates 247 (Brownfield et al., 2013; Gjorevski et al., 2015). This suggested that collagen 248 became increasingly bundled and condensed where aggregates were elongating.

Increased collagen bundling was also evident at the early stages of elongation. Because aggregates varied in the timing of when they broke symmetry, we examined aggregates by live imaging in the first 3 days of the assays. We then subdivided these based on their symmetry ratio, and compared the coherency of collagen 1 around the elongating areas of aggregates with that around the nonelongating areas (i.e. those that remained rounded, expressed as a fold-difference). This showed that collagen 1 coherency increased around the elongating areas as they broke symmetry (Figure 2E). Thus, from the early stages of symmetry breaking collagen 1 bundling increased around the sites where aggregates elongated.

Collagen bundling required cell proliferation, as coherency was reduced by treatment with either mitomycin $\mathrm{C}$ or aphidicolin (Figure 2F,G). Collagen bundling was also reduced when we inhibited cellular contractility with the myosin antagonist, blebbistatin, or the Rho kinase (ROCK) inhibitor, Y27632 (Figure 2H,I), consistent 262 with reports that cell contractility can condense collagen (Brownfield et al., 2013; 263 Buchmann et al., 2021; Gjorevski et al., 2015). In contrast, collagen condensation 264 persisted around aggregates treated with the Rac1 GEF inhibitor NSC23766, which 
265 did not affect aggregate elongation or the speed with which cells moved within the

266 aggregates (Figure 2-figure supplement 2). This suggested that aggregates exerted

267 forces to reorganize their local collagen environment as they broke symmetry.

\section{Collagen polarity coaligns with cell aggregates during elongation.}

We then asked if higher order organization in the collagen 1 gels was altered as aggregates elongated. For this, we used Orientation $\mathrm{J}$ to extract the principal axis of orientation in the gel, as a measure of its polarity, around elongating or nonelongating parts of an aggregate. Then we compared these gel orientations with the axis of elongation of the cell aggregate. First, we assembled histograms of gel orientation, setting the principal axis of elongation in the whole aggregate as $0^{\circ}$ (Figure 2J). The gels around non-elongating areas of the aggregates showed a broad distribution of orientations relative to the axis of the aggregate (Figure $2 \mathrm{~J}$ ). In contrast, the gel around the elongating regions of aggregates tended to orient with the principal axis of the aggregates. This suggested that the gel preferentially coaligned with the aggregates around the regions of elongation. This notion was reinforced by comparing the angle differences between the principal axis (polarity) of the gel and of the aggregates. In this analysis, a decrease in the difference in angles between these two axes indicates an increase in their co-alignment. We found that the angle differences between the axes of collagen polarity and aggregate elongation was significantly smaller around the elongating regions than around the non-elongating regions of the aggregates (Figure $2 \mathrm{~K}$ ). Thus, the collagen matrix became polarized and co-aligned with the aggregates around regions of elongation.

Collagen polarization stimulates cell proliferation to induce aggregate elongation.

This led us to wonder if polarization of the collagen could itself influence the process of aggregate elongation. To test this, we developed a strategy that applied exogenous stretch to polarize the matrix independently of cell-generated forces. In this protocol, a collagen gel was created within a ring-shaped polydimethylsiloxane (PDMS) frame, then stretched uniaxially by expanding a stretcher inserted into the hole of the ring for $4 \mathrm{~h}$ (Figure 3-figure supplement 1A,B). Second harmonic imaging confirmed that collagen fibers were isotropically distributed in the unstretched gels and became more coherent (bundled) and more polarized immediately after stretching (Figure 3-figure supplement 1C,D), with their principal axes oriented in the direction of stretch (Figure 3-figure supplement 1E). Moreover, polarization of the collagen fibrils could be preserved for at least 7 days after the application of stretch by re-embedding the collagen rings in a larger collagen gel (Figure 3A-C, Figure 3-

305 figure supplement 1B). In contrast, when gels were allowed to float in medium after 306 stretching (Figure 3D, Figure 3-figure supplement 1B), gel coherency (Figure 3E) 307 and polarity (Figure $3 F$ ) reverted to that of unstretched gels. Thus, reembedding 
308 allowed us to sustain collagen polarization for a prolonged period after the initial 309 stretching.

310 We then transplanted spherical cell aggregates from Matrigel ${ }^{\mathrm{TM}}$ into the 311 collagen rings and applied our stretching protocol. Interestingly, the aggregates did 312 not become elongated during the period when stretch was actively applied. The 313 symmetry ratios of aggregates immediately after stretching were identical to those in 314 unstretched aggregates (Figure 3-figure supplement 2A,B). Instead, aggregates 315 broke symmetry many hours after the period of active stretching. However, 316 symmetry breaking occurred earlier when stretched gels were allowed to retain 317 collagen polarization by re-embedding $(35.14 \pm 3.28 \mathrm{~h}$, Figure $3 \mathrm{G}, \mathrm{H})$ compared with 318 aggregates that were allowed to break symmetry spontaneously in non-stretched 319 gels $(68.40 \pm 5.88 \mathrm{~h}, \mathrm{p}<0.001)$. Moreover, the degree of elongation was also 320 enhanced once it had begun. The symmetry ratios of aggregates embedded in 321 stretch-polarized gels were significantly greater than those seen with native gels 322 (Figure 3I). Therefore, polarization of the collagen gel could stimulate the cell aggregates to break symmetry.

Stretch-polarized aggregates also elongated preferentially along the axis of the exogenous stretch, whereas aggregates in non-stretched gels elongated in a random direction (Figure 3G,J, Figure 3-figure supplement 2C,D; Video 2). The angle difference between collagen fiber polarity and aggregate elongation was significantly smaller in stretch-polarized gels than in unstretched control gels (Figure $3 \mathrm{~K})$. Of note, collagen gel reorganization was preserved in the stretch-polarized gels (Figure 3L,M), even in the presence of cellular aggregates, and this was oriented in the direction of the original stretch. This suggested that in this assay the elongating aggregates were following the polarity of the gel itself. In contrast, similar intensity of fibronectin staining was seen in stretch-polarized aggregates compared with controls, suggesting that fibronectin deposition was not stimulated by the stretch stimulation (Figure 3-figure supplement 2E).

Importantly, the impact of stretch on the aggregates was disrupted when collagen polarization was reversed. The proportion of aggregates that elongated after stretching was reduced when the gels were floated rather than being reembedded (Figure 4A) and their length of elongation was reduced (Figure 4B). Of note, cell-based forces could not overcome the external forces acting on the gel as the reversal of collagen polarization occurred even when aggregates were incorporated into the gel (Figure 4C). This implied that collagen polarization may have been responsible for allowing external stretch to promote aggregate elongation.

To confirm that these results were due to a critical role of collagen polarization, rather than as-yet-unknown impacts of stretch upon the cells, we used collagenase to extract aggregates from gels immediately after their $4 \mathrm{~h}$ stretch, then transplanted them into unstretched gels where the collagen fibers oriented randomly (Figure 4D). We reasoned that if elongation were due to a cell-intrinsic mechanism that bore the memory of the stretch, then this should be preserved even after cells were transplanted into a naïve gel. As noted earlier, aggregate elongation occurred earlier when stretched gels were reembedded. However, this effect was lost when cells 
352 were removed from the stretched gel immediately after stretching and transplanted

353 into a naïve isotropic gel (Figure 4E). Together, these results indicate that collagen

354 polarization can direct aggregate elongation, to accelerate its initiation and orient the 355 direction of symmetry-breaking.

\section{Collagen matrix polarization stimulates cell proliferation for anlagen elongation.}

359

Since cell proliferation was a major driver of spontaneous aggregate elongation, we then asked if it was altered when the collagen gel was polarized by stretch. Indeed, we found that stretch-polarization stimulated cell proliferation (Figure 5A). An increase in the proportion of Ki67-positive cells was evident at day 1 after stretchpolarization, whereas it did not increase till day 2 in native gels. Moreover, the proportion of Ki-67 cells was consistently greater in stretch-polarized gels during the early phase of culture (day 1-3) as well as at the end of our experiments (day 8). Cell proliferation mediated the enhanced elongation in the stretched gels, as both the length (Figure 5B) and symmetry ratio (Figure 5C) of stretch-stimulated aggregates were reduced by mitomycin $\mathrm{C}$ and aphidicolin.

However, proliferation was not increased if gels were allowed to float, rather than being re-embedded, after stretching. Floating the gels reduced the proportion of proliferating cells in the aggregates overall (Figure 5D,E) as well as those specifically within the area of elongation (Figure 5F), compared with gels that had been reembedded to preserve collagen polarization. This implied that it was sustained polarization of the gel, rather than simply transfer into the collagen environment, that could stimulate cell proliferation to elongate aggregates.

\section{Polarized collagen promotes cell proliferation via ERK pathway.}

Extracellular signal-regulated kinase $(E R K 1 / 2)$ is one of the major pathways that stimulates cell proliferation in mammary epithelial cells (Moreno-Layseca and Streuli, 2014; Streuli and Akhtar, 2009; Walker and Assoian, 2005). To evaluate its possible role in aggregate elongation, we expressed the ERK/KTR-mClover biosensor, which translocates out of the nucleus when ERK is activated (de la Cova et al., 2017). We confirmed the action of the sensor, as it accumulated in the nuclei of monolayer cultures that were treated with the ERK inhibitor FR180204 (50 $\mu \mathrm{M})$ (Figure 6-figure supplement 1A). Using this sensor in 3D cultures, we found that the proportion of ERK-activated cells was significantly greater in the elongating regions of aggregates that had spontaneously broken symmetry, than in the non-elongating areas (Figure 3906 A, B). This was especially apparent in cells at the surface of aggregates that were 391 in contact with the collagen gel (Figure 6C). In contrast, although Hippo pathway 392 signaling has been implicated in breaking the symmetry of epithelial organoids 393 (Serra et al., 2019), we did not detect any changes in nuclear Yap1 staining that 394 might indicate that this pathway was being activated in our experiments (Figure 6- 
395 figure supplement $1 \mathrm{~B}, \mathrm{C})$. This suggested that ERK $1 / 2$ signaling might be a 396 candidate for collagen polarization to stimulate cell proliferation.

This notion was supported by finding that FR180204 $(50 \mu \mathrm{M})$ reduced cell proliferation in response to stretch-polarization (Figure 6D,E) and decreased the proportion of aggregates that elongated (Figure 6F). As well, the length (Figure 6G) and asymmetric morphology (Figure $6 \mathrm{H}$ ) were reduced in those aggregates that did elongate. Thus, ERK1/2 appeared to be critical for collagen polarization to stimulate

402

403 cell proliferation for aggregate elongation.

\section{Integrins are necessary for polarized collagen to stimulate elongation.}

Integrins are the major ECM receptors in the mammary gland and other epithelia, and they control diverse aspects of cellular function, including cell proliferation (Miranti and Brugge, 2002; Wozniak et al., 2003). We therefore asked if integrin signaling might have stimulated cell proliferation and aggregate elongation in response to collagen polarization (Figure $7 \mathrm{~A}$ ).

We focused on the $\beta 1$-integrins which have been implicated in regulating cell proliferation during mammary gland development (Li et al., 2005). This class of integrins contains a number of collagen 1 receptors, including $\alpha 2 \beta 1$-integrin (Heino, 2000; Käpylä et al., 2000) which was expressed in our MCF10A cells (Figure 7B). Then we used the inhibitory AllB2 antibody to block $\beta 1$-integrin during stretchpolarization experiments. mAb AllB2 $(15 \mu \mathrm{g} / \mathrm{ml})$ was added as aggregates were transplanted into the collagen gel rings and replenished after 7 days. mAb AIIB2 blocked the induction of proliferation in stretch-polarized gels, the proportion of Ki67positive cells being significantly reduced in AllB2-treated cultures compared with controls (Figure 7C,D). This was accompanied by inhibition of elongation. After 3 days incubation, $49.19 \pm 1.61 \%$ aggregates started to elongate in controls, whereas less than $20 \%$ of aggregates elongated when $\beta 1$-integrins were inhibited (AllB2: $17.86 \pm 4.78 \%$ ) (Figure 7E). Moreover, even when mAb AllB2-treated aggregates eventually elongated upon prolonged culture (comparing the proportion of elongated cells at 7 days with that at 3 days), these aggregates remained shorter (Figure 7F) and less asymmetric (Figure 7G) than in the absence of AllB2. Finally, we asked if the ERK response required $\beta 1$-integrins. Indeed, we found that the ability of stretchpolarization to stimulate ERK signaling at early (day 2) and later stages (day 7) was reduced when $\beta 1$-integrins were blocked with mAb AllB2 (Figure $7 \mathrm{H}, \mathrm{I}$ ). This implied that a $\beta 1$-integrin-ERK pathway was responsible for stimulating epithelial proliferation.

\section{Discussion}

These results lead us to conclude that a polarized collagen 1 matrix can promote epithelial elongation by stimulating locoregional cell proliferation. We infer this because: 1) Loco-regional cell proliferation was a striking feature of MCF10A elongation in our experiments, whose loss was not compensated for by other 
439 morphogenetic processes. 2) The pattern of proliferation within aggregates was 440 critically influenced by the polarized organization of the surrounding collagen 1 441 matrix. Together, we suggest that these reveal an interplay between collagen polarization and cell proliferation that can guide epithelial elongation (Figure 8).

Cell proliferation was a distinguishing feature of the elongation process in our MCF10A model. Increased proliferation was observed in elongating aggregates and, indeed, began to increase before elongation was detected. Furthermore, proliferation was greater in the regions of the aggregates that underwent elongation compared to those areas that did not. This suggested that a loco-regional increase in cell proliferation might be important for the elongation process, a notion that was supported when elongation was inhibited by blocking proliferation. Elongation was reduced when mitomycin $\mathrm{C}$ and aphidicolin were added from the beginning of the assays, consistent with earlier evidence that inhibiting proliferation early in the culture process blocked branching morphogenesis in mouse mammary organoids (Ewald et al., 2008). We also found that elongation was compromised if proliferation was inhibited after the elongation process had begun, a later dependence that was not seen in organoids (Huebner et al., 2016). Differences in cell system may therefore influence the impact of cell proliferation on elongation. Nonetheless, our data strongly suggest that loco-regional stimulation of cell proliferation was required for epithelial elongation in our model.

This raised the question of how cell proliferation might be preferentially increased within specific regions of MCF10A aggregates. Several of our observations implicate polarized reorganization of the collagen 1 matrix in this specification process. First, loco-regional polarization of the collagen 1 matrix accompanied aggregate elongation when cell aggregates spontaneously broke symmetry. This reorganization was distinguished by increased coherency of the matrix, consistent with an increase in collagen bundling, as well as polarization of the gel so that it became oriented with the axis of the elongating aggregate. Matrix reorganization concentrated where the aggregates were elongating and proliferation, as has been reported earlier (Brownfield et al., 2013; Buchmann et al., 2021; Gjorevski et al., 2015). Second, aggregate elongation was stimulated when the gel was polarized by applying an external stretch. Similarly, collagen 1 orientation has been reported to mammary organoid branching (Brownfield et al., 2013). But the stimulatory effect on elongation was lost if the stretched gels were allowed to lose their polarization or if aggregates were transplanted into a naïve, unstretched gel. This implied that the cells were responding to the altered organization of the gel, rather than simply exposure to collagen 1. Consistent with what we had observed when aggregates spontaneously broke symmetry, stretch-induced polarization of gels stimulated cell proliferation and this was necessary for the accelerated elongation process to occur. This appeared to be mediated by a $\beta 1$-integrin and ERK-dependent pathway. enhance their proliferation? The polarization of collagen networks can have complex effects on cells. Increasing fibril alignment in acellular collagen gels was reported to 
483 increase fibre stiffness and decrease pore size (Riching et al., 2014; Taufalele et al., 484 2019), even in the absence of externally applied forces. Moreover, in single cell 485 cultures aligned collagen fibres were associated with larger focal adhesions than 486 non-aligned fibrils (Doyle and Yamada, 2016). One possibility, then, is that integrin 487 signaling was responding to the increased stiffness of the polarized collagen gels. 488 This is consistent with the well-characterized role for integrins to sense changes in 489 matrix stiffness (Giannone and Sheetz, 2006). As well, collagen 1 can bind to, and 490 sequester, a variety of growth factors. Whether this reservoir can be released when 491 cells apply tension to collagen is an interesting question for further consideration 492 (Wipff and Hinz, 2008).

493 Cell migration is a key driver of branching morphogenesis (Gjorevski et al., 494 2015; Huebner et al., 2016) and earlier experiments reported that the application of 495 external stretch to collagen-embedded aggregates could promote elongation through 496 cell migration (Brownfield et al., 2013). We, too, observed cell migration in our 497 aggregates and, although their speeds were not different, migrating cells within 498 elongating parts of the aggregates tended to align with the axis of elongation and 499 move slightly more persistently than cells moving within the non-elongating regions 500 of the aggregates. These features of collective migration might be expected to 501 contribute to aggregate elongation.

$502 \quad$ Interestingly, blocking cell proliferation decreased the apparent persistence of 503 the migrating cells and their alignment with the principal axes of the aggregates. 504 Therefore, rather than collective migration being enhanced to compensate for the 505 decrease in proliferation, cell proliferation appeared to support these elongation506 facilitating aspects of cell migration. One possible explanation is that persistence and 507 directional alignment were being guided by the reorganized collagen which, in effect, 508 served to create a 3-dimensional micropattern around the aggregates. Studies in 2509 dimensional systems have shown that cells can orient their patterns of migration 510 when collagen networks condense and form bundles (Mohammed et al., 2020; Wang 511 et al., 2018). As collagen reorganization was reduced by inhibiting proliferation, 512 mitomycin $\mathrm{C}$ and aphidicolin might have affected cell migration indirectly through 513 matrix organization. Such micropatterning might also influence other aspects of cell 514 behaviour during elongation. Micropatterning experiments using 2-dimensional 515 substrata have shown that anisotropic confinement can orient patterns of cell division 516 (Thery et al., 2005), potentially by orienting tensile stresses (Campinho et al., 2013; 517 Legoff et al., 2013), as would also be predicted to accompany stiffer, condensed 518 collagen bundles. Consistent with this, we observed that cells within the elongating 519 areas appeared to orient their divisions with the axis of elongation, an effect which 520 would also be predicted to enhance elongation. These observations reinforce the 521 notion that interplay between cell proliferation and collagen (re)organization is a key 522 contributor to epithelial elongation.

$523 \quad$ The reductionist model that we used in these experiments allowed us to focus 524 on testing how collagen organization could promote epithelial elongation. How might 525 the results from this system operate in the more complex environment of the tissue?

526 One important factor to consider is the basement membrane, which can separate the 
527 epithelial cell compartment from collagen 1 in the stroma. However, earlier studies

528 reported that the basement membrane thins substantially and is remodelled in

529 regions of epithelial elongation, such as the tips of mammary or salivary gland buds

530 (Harunaga et al., 2014; Silberstein and Daniel, 1982; Williams and Daniel, 1983),

531 potentially allowing elongating regions of epithelia to engage with collagen 1.

532 Evaluating how cell proliferation, stromal collagen organization are coordinated with

533 other components of the ECM in physiological models of developing glands will be

534 an important question for future research.

535 In conclusion, we propose the following working model (Figure 8): Regional

536 polarization of the collagen 1 matrix begins in response to anisotropies in force that

537 aggregates exert upon their ECM via their integrin adhesions (Figure 7A). Cell-based

538 forces exerted through integrins can apply strain on collagen fibrils (Brownfield et al.,

539 2013; Buchmann et al., 2021; Gjorevski et al., 2015; Hall et al., 2016) and, consistent

540 with this, collagen reorganization in our experiments was compromised by inhibiting

541 cellular contractility. However, compression of collagen by increasing cell numbers or

542 cell movements could also have contributed (Buchmann et al., 2021). By implication,

543 collagen polarization will be greater around the parts of the aggregate that are

544 generating more force. The consequent loco-regional collagen polarization then

545 stimulates further cell proliferation in the adjacent parts of the aggregate to sustain

546 elongation. It was interesting to note that collagen reorganization was compromised

547 when cell proliferation was blocked from the outset of the assays. Possibly, subtle

548 differences in cell proliferation may have contributed to generating initial anisotropies

549 in force to reorganize the gels. If so, we speculate that the increase in cell

550 proliferation may have contributed to further collagen reorganization, with the

551 capacity to develop a feedback system that might help sustain aggregate elongation.

552 This would allow polarized collagen networks to effectively provide a structural

553 memory of the initial axis of symmetry breaking, i.e., a relatively long-lived spatial

554 cue that directs further elongation of the epithelial anlagen.

\section{Acknowledgements}

558

559

560

561

562

563

564

565

566

We thank Selwin Wu for advice and all our lab colleagues for their feedback and support. The authors were supported by the National Health and Medical Research Council of Australia (Fellowship 1136592 and GNT1123816, 1140090 to ASY), Australian Research Council (DP19010287, 190102230 to AY and FT190100516 to SS), a Snow Medical Fellowship to $\mathrm{JH}$, and a postdoctoral fellowship from The Uehara Memorial Foundation to HK.

\section{Materials and Methods}

\section{Cell culture and lentivirus infection}

MCF10A human mammary epithelial cells were cultured in DMEM/F12 medium

571 supplemented with $5 \%$ horse serum, $10 \mu \mathrm{g} / \mathrm{ml}$ insulin, $0.5 \mu \mathrm{g} / \mathrm{ml}$ hydrocortisone, 100 
$572 \mathrm{ng} / \mathrm{ml}$ cholera toxin, $20 \mathrm{ng} / \mathrm{ml}$ EGF, 100 units $/ \mathrm{ml}$ Penicillin and 100 units $/ \mathrm{ml}$

573 Streptomycin as previously described (Debnath et al., 2002).

574 HEK-293T cells were transfected using Lipofectamine 2000 (Invitrogen) for lentiviral

575 expression vector pLL5.0 and third generation packaging constructs pMDLg/pRRE,

576 RSV-Rev and pMD.G. Third generation packaging constructs were kindly provided

577 by Prof. James Bear (UNC Chapel Hill, North Carolina, USA). After transducing

578 MCF10A cells with either pLL5.0-EGFP-HRasC20, pLL5.0-NLS-mCherry or pLenti-

579 ERK/KTR-mClover lentivirus construct, we isolated highly-expressing cells using

580 Fluorescence-activated cell sorting (Influx Cell sorter; Cytopeia).

581

582

\section{D Matrigel and collagen culture}

584 Matrigel (FAL354230; Corning) and overlayed with Matrigel including medium in 8 585 well chamber coverglass (0030742036; Eppendorf) as previously described 586 (Debnath et al., 2002; Debnath et al., 2003). In brief, single isolated MCF10A cells 587 were mixed with an assay medium containing $2 \%$ Matrigel, $5 \mathrm{ng} / \mathrm{ml}$ EGF and seeded 588 on a solidified layer of $100 \%$ Matrigel at 10000 cells/well. After seeding cells on Matrigel, medium was changed every 4 days.

In collagen culture, single isolated MCF10A cells were mixed with collagen gel at 20000 cells/well (FAL354236; Corning). This mixture was neutralized with $\mathrm{NaOH}$ and HEPES buffer and collagen concentration was adjusted to $1.0 \mathrm{mg} / \mathrm{ml}$ with culture medium on ice. The $\mathrm{pH}$ of collagen solution was checked by litmus paper. Collagen solution with cells was seeded in 8 well chamber coverglass and solidified at $37 \square$ for $30 \mathrm{~min}$.

\section{Transplantation of MCF10A aggregates}

MCF10A aggregates cultured on Matrigel for 10 days were washed with PBS and incubated with cold cell recovery solution (FAL354253; Corning) for $30 \mathrm{~min}$ on ice. Aggregates were collected into a tube containing the cell recovery solution, spun down at $1200 \mathrm{rpm}$, and washed with cold PBS. PBS-washed aggregates were resuspended in MCF10A culture medium and mixed with collagen solution neutralized with $\mathrm{NaOH}$ and HEPES buffer. Collagen gel solution with aggregates were solidified at $37 \square$ for $30 \mathrm{~min}$.

Aggregates embedded in collagen gel were isolated from gel by dissolving the gel with collagenase (C2799; Sigma). Gels were washed with PBS and incubated in 20 $\mu \mathrm{g} / \mathrm{ml}$ collagenase in Hanks' Balanced Salt Solution (H8264; Sigma) at $37 \square$ for 30 min to dissolve the collagen gel. Aggregates were collected with incubated solution into the tube and mixed with DMEM/F12 medium which contains $20 \%$ horse serum. Solution was spun down at $1200 \mathrm{rpm}$ and pellet was washed with PBS. PBS-washed aggregates were resuspended in culture medium and re-embedded in collagen gel.

\section{Plasmids}

pLL5.0-NLS-mCherry was constructed in previous study (Leerberg et al., 2014). pLent-ERK/KTR-mClover was obtained from addgene (\#59150). pLL5.0-EGFPHRasC20 was generated by insertion of EGFP-HRasC20 fragment which was amplified from pEGFP-F (\#6074-1; Clontech) by PCR into pLL5.0 vector. pLL5.0EGFP (gift from Prof. James Bear; UNC Chapel Hill, North Carolina, USA) was digested with EcoRI and Sbfl to remove EGFP, and then EGFP-C20 fragment was inserted and ligated by In-Fusion cloning kit (638910; Clontech). 


\section{Antibodies and inhibitors}

623 Primary antibodies used for immunocytochemistry in this study were rabbit anti-Ki67 624 (ab15580; Abcam), mouse anti-laminin V (MAB19562; Chemicon), rat anti-E625 cadherin (131900; Invitrogen), mouse anti-GM130 (610822; BD), rabbit anti626 fibronectin (F3648; Sigma) and rabbit anti-YAP1 (4912; Cell Signaling Technology). 627 F-actin was stained with AlexaFluor 488-, 594-, 647-phalloidin (Invitrogen). Primary 628 antibodies used for immunoblot were rabbit anti-integrin a2 (ab181548; Abcam), 629 mouse anti-integrin $\beta 1$ (610467; BD Transduction Laboratories) and rabbit anti630 p44/42 MAPK (Erk1/2) (9102; Cell Signaling Technology). AllB2 antibody for 631 blocking integrin $\beta 1$ was purchased from Developmental Studies Hybridoma Bank 632 and treated at $15 \mu \mathrm{g} / \mathrm{ml}$ in this study.

633 Proliferation inhibitors mitomycin C (M7949; Sigma) and aphidicolin (178273; Merck) 634 were used at $10 \mu \mathrm{M}$ and $2 \mu \mathrm{M}$ respectively. Myosin II inhibitor blebbistatin (203390; 635 Sigma) and ROCK inhibitor Y-27632 (688000; Sigma) were used at $25 \mu \mathrm{M}$ and 30 $636 \mu \mathrm{M}$ respectively. ERK1/2 inhibitor FR180204 (SC-203945; Santa Cruz) was used at $63750 \mu \mathrm{M}$. Except for mitomycin C, cell aggregates were treated with the inhibitors after 638 transplantation into collagen gels. Inhibitors were then replenished when medium was changed and left in the collagen culture until cell aggregates were ready to be fixed. For mitomycin $\mathrm{C}$ wash out experiments, cell aggregates were treated with mitomycin $\mathrm{C}$ for 1 hour, then washed 3 times with PBS and left to recover in MCF10A culture medium.

\section{Immunocytochemistry and microscopy}

3D cultured cells in gel were fixed with $4 \%$ paraformaldehyde in cytoskeleton stabilization buffer $(10 \mathrm{mM}$ PIPES at $\mathrm{pH} 6.8,100 \mathrm{mM} \mathrm{KCl}, 300 \mathrm{mM}$ sucrose, $2 \mathrm{mM}$ EGTA and $2 \mathrm{mM} \mathrm{MgCl}$ ) at room temperature for $30 \mathrm{~min}$, followed by a treatment for 30 min with $0.5 \%$ TritonX-100 and $10 \%$ goat serum in PBS for 1 hour at room temperature. Then they were stained with primary antibodies for overnight at $4 \square$. Subsequently, cells were washed with PBS and incubated with secondary antibodies with phalloidin and DAPI for 1 hour at room temperature.

Confocal images were acquired with an upright Meta laser scanning confocal microscope (LSM710; Zeiss) equipped with plan-Apochromat 20x 0.8NA or 40x 1.3NA objectives (Zeiss) and zen2012 software (Zeiss). The fluorescent images of collagen fibrils probed with mCherry-CNA35 were acquired by inverted microscope (Ti2; Nikon) equipped with Dragonfly spinning disc (Andor), by using plan-Apo 10x $0.45 \mathrm{NA}, 20 \mathrm{x}$ 0.75NA or 40x 0.95NA dry objectives (Nikon) and Fusion software (Andor).

Fluorescent Images of the SHG signal from collagen-1 were collected with an inverted confocal microscope (LSM710; Zeiss) equipped with multiphoton laser by using $860 \mathrm{~nm}$ excitation with SHG signal obtained with $690 \mathrm{~nm}$ bandpass filter. A 40x 1.3NA or $63 \times 1.40 \mathrm{NA}$ plan-apochromat oil objectives (Zeiss) were used to obtain SHG signals.

Fluorescence and phase contrast live images of elongating aggregates were acquired with an inverted fluorescence microscope (IX81; Olympus) equipped with CCD-camera (Hamamatsu) and an incubation box (Clear State Solutions) maintained at $37 \square$ and $5 \% \mathrm{CO}_{2}$ with gas controller (OkoLab), using plan-Apo 10x $0.4 \mathrm{NA}$ objective (Olympus) and CellSens software (Olympus).

\section{Collagen gel labeling and stretching}


671 Collagen fibrils were labelled with mCherry-CNA35 by mixing with purified mCherry-

672 CNA35 protein at $2 \mu \mathrm{M}$ before gelling. Gels were solidified at $37 \square$ for $30 \mathrm{~min}$.

673 PDMS gel frame and stretchers for gel stretching were kindly gifted from Dr. James

674 Hudson (QIMR Berghofer, Queensland, Australia). $1.5 \mathrm{mg} / \mathrm{ml}$ collagen gels were

675 solidified as a ring-shape in PDMS frame. PDMS stretchers were inserted into the

676 hole of the gel and expanded, and incubated for 4 hours in culture medium.

677 Stretched gels were released and then floated in medium or re-embedded in

678 collagen gel. For re-embedding, stretched gels were put in $1.5 \mathrm{mg} / \mathrm{ml}$ collagen gel

679 and solidified at at $37 \square$ for $30 \mathrm{~min}$.

680

681

682

683

684

685

686

687

688

689

690

691

692

\section{CNA35-mcherry protein purification}

Protein expression vector pET28a-mCherry-CNA35 was obtained from Addgene (\#61607). Transformed E.coli (BL21) was cultured in $400 \mathrm{ml} \mathrm{LB}$, and induced protein expression with isopropyl $\beta-D-1$-tthiogalactopyranoside for 20 hours at $25 \square$. Cultured bacteria were spun down, and collected bacteria pellet was sonicated. The lysate was centrifuged and the resulting supernatant was run through a column filled with N-NTA His-band resin (Millipore). Bound protein was eluted and then dialyzed for overnight in PBS at $4 \square$. Endotoxin was removed by endotoxin removal columns (88274; Thermo) following with manufacturer's protocol.

\section{Quantitative analysis of collagen fibril alignment and aggregates elongation}

Fluorescent confocal images of collagen fibrils acquired SHG microcopy or CNA35 probes were used for collagen fibril analysis. We analysed collagen organization in these images using Orientation $\mathrm{J}$ which calculates the local orientation and isotropy for each pixel in an image based on the structure tensor for that pixel (Rezakhaniha et al., 2012). The structure tensor is evaluated for each pixel of the given image by calculating the spatial partial derivatives by using (a cubic B-spline) interpolation. The local orientation and isotropy for each pixel are computed based on the eigenvalues and eigenvectors of the structure tensor. We characterized three features in the organization of collagen fibrils orientations (Clemons et al., 2018): i) The coherency of fibrils, defined as co-orientation in the same direction, as a measure of bundling. ii) Isotropy, the distribution of fibril orientation in the field of analysis; and iii) Polarization, defined as the principal axis of fibril orientation in anisotropic gels, relative to a reference axis. $50 \times 50$ um or $100 \times 100$ um size of $\mathrm{ROI}$ were selected from SHG images or CNA35 probed images respectively and used for analysis. Elongation axis of aggregates were measured by the angle tool in ImageJ, and then we calculated the angle difference between principal axis of collagen fibrils or stretching axis. The fold difference of gel coherency was measured by dividing the coherency of fibrils in the elongating area by the non-elongating area. The symmetry ratio of aggregates was measured by dividing the longest length by widest width of aggregates.

\section{Quantitative analysis of proliferating cells and nuclear division angle}

The number of cells co-stained with DAPI and anti-Ki67 antibody were counted by Imaris software (Bitplane). The number of Ki67 positive cells were divided by the total number of nucleus stained with DAPI to calculate percentage of Ki67 positive cells. For live imaging, cells expressed with NLS-mCherry to count their number. The frequency and orientation of cell division were analyzed from the time lapse images by ImageJ. We set the elongation axis of aggregate as a reference and measured the angle difference between elongation axis and dividing axis of nucleus. 


\section{Analysis of nuclear tracking}

To obtain the migration speed of cells, we tracked individual cell nuclei using the Spot function in the Imaris software (Huebner et al., 2016). The fluorescent images of cells expressing NLS-mCherry were acquired every 10 minutes for at least 50 hours. To obtain the track displacement angle, we first calculated the displacement angle of nuclei from the displacement of $X$ and $Y$ axis between first and last position, and then measured the angle difference from elongating angle of aggregates. Track straightness was calculated from track displacement by track length. Inhibitors were treated 1 hour before the imaging.

\section{Quantitative analysis of ERK/KTR biosensor}

ERK activity was judged by the location of mClover fluorescent signal in individual cells. Briefly, fluorescent tagged KTRs translocate between nuclei and cytoplasm depends on kinase activity. When ERK activity is high, KTR-mClover should localize in cytoplasm (de la Cova et al., 2017). Aggregates expressing ERK/KTR-mClover were transplanted into collagen gel, and were co-stained with phalloidin and DAPI after fixation. To judge the delocalization of KTR-mClover, we used line intensity scan in single plane images and then manually counted ERK active cells.

\section{Statistical analysis}

Significance was determined by unpaired Student's t test and one-way ANOVA by using GraphPad Prism 8 (GraphPad software).

\section{References}

Affolter, M., Zeller, R., and Caussinus, E. (2009). Tissue remodelling through branching morphogenesis. Nat Rev Mol Cell Biol 10, 831-842. 10.1038/nrm2797.

Andrew, D.J., and Ewald, A.J. (2010). Morphogenesis of epithelial tubes: Insights into tube formation, elongation, and elaboration. Dev Biol 341, 34-55. 10.1016/j.ydbio.2009.09.024. Bastidas-Ponce, A., Scheibner, K., Lickert, H., and Bakhti, M. (2017). Cellular and molecular mechanisms coordinating pancreas development. Development 144, 2873-2888.

10.1242/dev.140756.

755 Brownfield, D.G., Venugopalan, G., Lo, A., Mori, H., Tanner, K., Fletcher, D.A., and Bissell, M.J.

756 (2013). Patterned collagen fibers orient branching mammary epithelium through distinct

757 signaling modules. Curr Biol 23, 703-709. 10.1016/j.cub.2013.03.032.

758 Buchmann, B., Engelbrecht, L.K., Fernandez, P., Hutterer, F.P., Raich, M.K., Scheel, C.H., and

759 Bausch, A.R. (2021). Mechanical plasticity of collagen directs branch elongation in human

760 mammary gland organoids. Nat Commun 12, 2759. 10.1038/s41467-021-22988-2.

761 Campinho, P., Behrndt, M., Ranft, J., Risler, T., Minc, N., and Heisenberg, C.P. (2013).

762 Tension-oriented cell divisions limit anisotropic tissue tension in epithelial spreading during

763 zebrafish epiboly. Nat Cell Biol 15, 1405-1414. 10.1038/ncb2869.

764 Chung, S., and Andrew, D.J. (2008). The formation of epithelial tubes. Journal of Cell Science

765

766

767 121, 3501-3504. 10.1242/jcs.037887.

Clemons, T.D., Bradshaw, M., Toshniwal, P., Chaudhari, N., Stevenson, A.W., Lynch, J., Fear, M.W., Wood, F.M., and lyer, K.S. (2018). Coherency image analysis to quantify collagen

768 architecture: implications in scar assessment. Rsc Adv 8, 9661-9669. 10.1039/c7ra12693j. 
769 Costantini, F., and Kopan, R. (2010). Patterning a complex organ: branching morphogenesis

770

771

772

773

774

775

776

777

778

779

780

781

782

783

784

785

786

787

788

789

790

791

792

793

794

795

796

797

798

799

800

801

802

803

804

805

806

807

808

809

810

811

812

813

814 and nephron segmentation in kidney development. Dev Cell 18, 698-712.

10.1016/j.devcel.2010.04.008.

de la Cova, C., Townley, R., Regot, S., and Greenwald, I. (2017). A Real-Time Biosensor for

ERK Activity Reveals Signaling Dynamics during C. elegans Cell Fate Specification. Dev Cell 42, 542-553 e544. 10.1016/j.devcel.2017.07.014.

Debnath, J., Mills, K.R., Collins, N.L., Reginato, M.J., Muthuswamy, S.K., and Brugge, J.S. (2002). The role of apoptosis in creating and maintaining luminal space within normal and oncogene-expressing mammary acini. Cell 111, 29-40.

Debnath, J., Muthuswamy, S.K., and Brugge, J.S. (2003). Morphogenesis and oncogenesis of MCF-10A mammary epithelial acini grown in three-dimensional basement membrane cultures. Methods 30, 256-268.

Doyle, A.D., and Yamada, K.M. (2016). Mechanosensing via cell-matrix adhesions in 3D microenvironments. Exp Cell Res 343, 60-66. 10.1016/j.yexcr.2015.10.033.

Economou, A.D., Brock, L.J., Cobourne, M.T., and Green, J.B. (2013). Whole population cell analysis of a landmark-rich mammalian epithelium reveals multiple elongation mechanisms. Development 140, 4740-4750. 10.1242/dev.096545.

Ewald, A.J., Brenot, A., Duong, M., Chan, B.S., and Werb, Z. (2008). Collective epithelial migration and cell rearrangements drive mammary branching morphogenesis. Dev Cell 14, 570-581. 10.1016/i.devcel.2008.03.003.

Frantz, C., Stewart, K.M., and Weaver, V.M. (2010). The extracellular matrix at a glance. J Cell Sci 123, 4195-4200. 10.1242/jcs.023820.

Giannone, G., and Sheetz, M.P. (2006). Substrate rigidity and force define form through tyrosine phosphatase and kinase pathways. Trends in Cell Biology 16, 213-223.

https://doi.org/10.1016/i.tcb.2006.02.005.

Gjorevski, N., and Nelson, C.M. (2010). Branch formation during organ development. Wiley Interdiscip Rev Syst Biol Med 2, 734-741. 10.1002/wsbm.96.

Gjorevski, N., Piotrowski, A.S., Varner, V.D., and Nelson, C.M. (2015). Dynamic tensile forces drive collective cell migration through three-dimensional extracellular matrices. Sci Rep 5, 11458. 10.1038/srep11458.

Gong, Y., Mo, C., and Fraser, S.E. (2004). Planar cell polarity signalling controls cell division orientation during zebrafish gastrulation. Nature 430, 689-693. 10.1038/nature02796.

Goodwin, K., and Nelson, C.M. (2020). Branching morphogenesis. Development 147. 10.1242/dev.184499.

Graham, M.F., Diegelmann, R.F., Elson, C.O., Lindblad, W.J., Gotschalk, N., Gay, S., and Gay, R. (1988). Collagen content and types in the intestinal strictures of Crohn's disease.

Gastroenterology 94, 257-265. 10.1016/0016-5085(88)90411-8.

Guo, C.L., Ouyang, M., Yu, J.Y., Maslov, J., Price, A., and Shen, C.Y. (2012). Long-range mechanical force enables self-assembly of epithelial tubular patterns. Proc Natl Acad Sci U S A 109, 5576-5582. 10.1073/pnas.1114781109.

Hall, M.S., Alisafaei, F., Ban, E., Feng, X., Hui, C.-Y., Shenoy, V.B., and Wu, M. (2016). Fibrous nonlinear elasticity enables positive mechanical feedback between cells and ECMs.

Proceedings of the National Academy of Sciences 113, 14043-14048. 10.1073/pnas.1613058113.

Harunaga, J., Hsu, J.C., and Yamada, K.M. (2011). Dynamics of salivary gland morphogenesis. J Dent Res 90, 1070-1077. 10.1177/0022034511405330. 
815 Harunaga, J.S., Doyle, A.D., and Yamada, K.M. (2014). Local and global dynamics of the 816 basement membrane during branching morphogenesis require protease activity and 817 actomyosin contractility. Dev Biol 394, 197-205. 10.1016/j.ydbio.2014.08.014.

818 Heino, J. (2000). The collagen receptor integrins have distinct ligand recognition and 819 signaling functions. Matrix Biology 19, 319-323. https://doi.org/10.1016/S0945-

$820 \quad$ 053X(00)00076-7.

821 Huebner, R.J., Neumann, N.M., and Ewald, A.J. (2016). Mammary epithelial tubes elongate

822 through MAPK-dependent coordination of cell migration. Development 143, 983-993.

823 10.1242/dev.127944.

824 Ingman, W.V., Wyckoff, J., Gouon-Evans, V., Condeelis, J., and Pollard, J.W. (2006).

825 Macrophages promote collagen fibrillogenesis around terminal end buds of the developing

826 mammary gland. Dev Dyn 235, 3222-3229. 10.1002/dvdy.20972.

827 Iruela-Arispe, M.L., and Beitel, G.J. (2013). Tubulogenesis. Development 140, 2851-2855.

828 10.1242/dev.070680.

829 Kanematsu, A., Marui, A., Yamamoto, S., Ozeki, M., Hirano, Y., Yamamoto, M., Ogawa, O.,

830 Komeda, M., and Tabata, Y. (2004). Type I collagen can function as a reservoir of basic

831 fibroblast growth factor. Journal of Controlled Release 99, 281-292.

832 https://doi.org/10.1016/j.jconrel.2004.07.008.

833 Käpylä, J., Ivaska, J., Riikonen, R., Nykvist, P., Pentikäinen, O., Johnson, M., and Heino, J.

834 (2000). Integrin alpha(2)I domain recognizes type I and type IV collagens by different

835 mechanisms. J Biol Chem 275, 3348-3354. 10.1074/jbc.275.5.3348.

836 Keely, P.J., Wu, J.E., and Santoro, S.A. (1995). The spatial and temporal expression of the

837 alpha 2 beta 1 integrin and its ligands, collagen I, collagen IV, and laminin, suggest important

838 roles in mouse mammary morphogenesis. Differentiation 59, 1-13. 10.1046/j.1432-

839 0436.1995.5910001.x.

840 Keller, R. (2002). Shaping the vertebrate body plan by polarized embryonic cell movements.

841 Science 298, 1950-1954.

842 Keller, R. (2006). Mechanisms of elongation in embryogenesis. Development 133, 2291-

843 2302. 10.1242/dev.02406.

844 Krahn, K.N., Bouten, C.V., van Tuijl, S., van Zandvoort, M.A., and Merkx, M. (2006).

845 Fluorescently labeled collagen binding proteins allow specific visualization of collagen in

846 tissues and live cell culture. Anal Biochem 350, 177-185. 10.1016/j.ab.2006.01.013.

847 Krause, S., Maffini, M.V., Soto, A.M., and Sonnenschein, C. (2008). A novel 3D in vitro culture

848 model to study stromal-epithelial interactions in the mammary gland. Tissue Eng Part $\mathrm{C}$

849 Methods 14, 261-271. 10.1089/ten.tec.2008.0030.

850 Leerberg, J.M., Gomez, G.A., Verma, S., Moussa, E.J., Wu, S.K., Priya, R., Hoffman, B.D.,

851 Grashoff, C., Schwartz, M.A., and Yap, A.S. (2014). Tension-sensitive actin assembly supports

852 contractility at the epithelial zonula adherens. Curr Biol 24, 1689-1699.

$85310.1016 /$ j.cub.2014.06.028.

854 Legoff, L., Rouault, H., and Lecuit, T. (2013). A global pattern of mechanical stress polarizes

855 cell divisions and cell shape in the growing Drosophila wing disc. Development 140, 4051-

856 4059. 10.1242/dev.090878.

857 Li, N., Zhang, Y., Naylor, M.J., Schatzmann, F., Maurer, F., Wintermantel, T., Schuetz, G.,

858 Mueller, U., Streuli, C.H., and Hynes, N.E. (2005). $\beta 1$ integrins regulate mammary gland

859 proliferation and maintain the integrity of mammary alveoli. The EMBO Journal 24, 1942-

860 1953. https://doi.org/10.1038/sj.emboj.7600674. 
861 Llacua, L.A., Faas, M.M., and de Vos, P. (2018). Extracellular matrix molecules and their 862 potential contribution to the function of transplanted pancreatic islets. Diabetologia 61, 863 1261-1272. 10.1007/s00125-017-4524-8.

864 Miranti, C.K., and Brugge, J.S. (2002). Sensing the environment: a historical perspective on 865 integrin signal transduction. Nature Cell Biology 4, E83-E90. 10.1038/ncb0402-e83.

866 Mohammed, D., Pardon, G., Versaevel, M., Bruyère, C., Alaimo, L., Luciano, M., Vercruysse, 867 E., Pruitt, B.L., and Gabriele, S. (2020). Producing Collagen Micro-stripes with Aligned Fibers 868 for Cell Migration Assays. Cellular and Molecular Bioengineering 13, 87-98. 10.1007/s12195869 019-00600-4.

870 Moreno-Layseca, P., and Streuli, C.H. (2014). Signalling pathways linking integrins with cell

871 cycle progression. Matrix Biology 34, 144-153.

872 https://doi.org/10.1016/i.matbio.2013.10.011.

873 Nakanishi, Y., Sugiura, F., Kishi, J., and Hayakawa, T. (1986). Collagenase inhibitor stimulates

874 cleft formation during early morphogenesis of mouse salivary gland. Dev Biol 113, 201-206.

875 10.1016/0012-1606(86)90122-3.

876 Nerger, B.A., and Nelson, C.M. (2018). 3D culture models for studying branching

877 morphogenesis in the mammary gland and mammalian lung. Biomaterials.

878 10.1016/j.biomaterials.2018.08.043.

879 Patel, V.N., Rebustini, I.T., and Hoffman, M.P. (2006). Salivary gland branching

880 morphogenesis. Differentiation 74, 349-364. 10.1111/j.1432-0436.2006.00088.x.

881 Piotrowski-Daspit, A.S., Nerger, B.A., Wolf, A.E., Sundaresan, S., and Nelson, C.M. (2017).

882 Dynamics of Tissue-Induced Alignment of Fibrous Extracellular Matrix. Biophys J 113, 702-

883 713. 10.1016/j.bpj.2017.06.046.

884 Price, R.G., and Spiro, R.G. (1977). Studies on the metabolism of the renal glomerular

885 basement membrane. Turnover measurements in the rat with the use of radiolabeled

886 amino acids. J Biol Chem 252, 8597-8602.

887 Rezakhaniha, R., Agianniotis, A., Schrauwen, J.T., Griffa, A., Sage, D., Bouten, C.V., van de

888 Vosse, F.N., Unser, M., and Stergiopulos, N. (2012). Experimental investigation of collagen

889 waviness and orientation in the arterial adventitia using confocal laser scanning microscopy.

890 Biomech Model Mechanobiol 11, 461-473. 10.1007/s10237-011-0325-z.

891 Riching, K.M., Cox, B.L., Salick, M.R., Pehlke, C., Riching, A.S., Ponik, S.M., Bass, B.R., Crone,

892 W.C., Jiang, Y., Weaver, A.M., et al. (2014). 3D collagen alignment limits protrusions to

893 enhance breast cancer cell persistence. Biophys J 107, 2546-2558.

894 10.1016/j.bpj.2014.10.035.

895 Schedin, P., and Keely, P.J. (2011). Mammary gland ECM remodeling, stiffness, and

896 mechanosignaling in normal development and tumor progression. Cold Spring Harb

897 Perspect Biol 3, a003228. 10.1101/cshperspect.a003228.

898 Schottenfeld, J., Song, Y., and Ghabrial, A.S. (2010). Tube continued: morphogenesis of the

899 Drosophila tracheal system. Curr Opin Cell Biol 22, 633-639.

900 https://doi.org/10.1016/i.ceb.2010.07.016.

901 Serra, D., Mayr, U., Boni, A., Lukonin, I., Rempfler, M., Challet Meylan, L., Stadler, M.B.,

902 Strnad, P., Papasaikas, P., Vischi, D., et al. (2019). Self-organization and symmetry breaking

903 in intestinal organoid development. Nature 569, 66-72. 10.1038/s41586-019-1146-y.

904 Shi, M., Zhu, J., Wang, R., Chen, X., Mi, L., Walz, T., and Springer, T.A. (2011). Latent TGF-

905 beta structure and activation. Nature 474, 343-349. 10.1038/nature10152. 
906 Shi, Q., Ghosh, R.P., Engelke, H., Rycroft, C.H., Cassereau, L., Sethian, J.A., Weaver, V.M., and

907 Liphardt, J.T. (2014). Rapid disorganization of mechanically interacting systems of mammary

908 acini. Proc Natl Acad Sci U S A 111, 658-663. 10.1073/pnas.1311312110.

909 Silberstein, G.B., and Daniel, C.W. (1982). Glycosaminoglycans in the basal lamina and

910 extracellular matrix of the developing mouse mammary duct. Dev Biol 90, 215-222.

911 10.1016/0012-1606(82)90228-7.

912 Simon-Assmann, P., Kedinger, M., De Arcangelis, A., Rousseau, V., and Simo, P. (1995).

913 Extracellular matrix components in intestinal development. Experientia 51, 883-900.

914 10.1007/BF01921739.

915 Soliman, N.A., and Yussif, S.M. (2016). Ki-67 as a prognostic marker according to breast

916 cancer molecular subtype. Cancer Biol Med 13, 496-504. 10.20892/j.issn.2095-

917 3941.2016.0066.

918 Sternlicht, M.D., Kouros-Mehr, H., Lu, P., and Werb, Z. (2006). Hormonal and local control of

919 mammary branching morphogenesis. Differentiation 74, 365-381. 10.1111/j.1432-

920 0436.2006.00105.x.

921 Streuli, Charles H., and Akhtar, N. (2009). Signal co-operation between integrins and other

922

923

924

925

926

927

928

929 receptor systems. Biochemical Journal 418, 491-506. 10.1042/bj20081948.

Taufalele, P.V., VanderBurgh, J.A., Munoz, A., Zanotelli, M.R., and Reinhart-King, C.A. (2019). Fiber alignment drives changes in architectural and mechanical features in collagen matrices. PLoS One 14, e0216537. 10.1371/journal.pone.0216537.

Thery, M., Racine, V., Pepin, A., Piel, M., Chen, Y., Sibarita, J.B., and Bornens, M. (2005). The extracellular matrix guides the orientation of the cell division axis. Nat Cell Biol 7, 947-953.

Tucker, A.S. (2007). Salivary gland development. Semin Cell Dev Biol 18, 237-244. 10.1016/j.semcdb.2007.01.006.

930 Varner, V.D., and Nelson, C.M. (2014). Cellular and physical mechanisms of branching

931 morphogenesis. Development 141, 2750-2759. 10.1242/dev.104794.

932 Verzijl, N., DeGroot, J., Thorpe, S.R., Bank, R.A., Shaw, J.N., Lyons, T.J., Bijlsma, J.W., Lafeber,

933 F.P., Baynes, J.W., and TeKoppele, J.M. (2000). Effect of collagen turnover on the

934 accumulation of advanced glycation end products. J Biol Chem 275, 39027-39031.

935 10.1074/jbc.M006700200.

936 Walker, J.L., and Assoian, R.K. (2005). Integrin-dependent signal transduction regulating

937 cyclin D1 expression and G1 phase cell cycle progression. Cancer and Metastasis Reviews 24, 938 383-393. 10.1007/s10555-005-5130-7.

939 Wang, W.Y., Pearson, A.T., Kutys, M.L., Choi, C.K., Wozniak, M.A., Baker, B.M., and Chen, C.S.

940 (2018). Extracellular matrix alignment dictates the organization of focal adhesions and

941 directs uniaxial cell migration. APL Bioeng 2, 046107. 10.1063/1.5052239.

942 Williams, J.M., and Daniel, C.W. (1983). Mammary ductal elongation: differentiation of

943 myoepithelium and basal lamina during branching morphogenesis. Dev Biol 97, 274-290.

944 10.1016/0012-1606(83)90086-6.

945 Wipff, P.J., and Hinz, B. (2008). Integrins and the activation of latent transforming growth

946 factor beta1 - an intimate relationship. Eur J Cell Biol 87, 601-615.

947 10.1016/j.ejcb.2008.01.012.

948 Wozniak, M.A., Desai , R., Solski, P.A., Der, C.J., and Keely , P.J. (2003). ROCK-generated

949 contractility regulates breast epithelial cell differentiation in response to the physical

950 properties of a three-dimensional collagen matrix. Journal of Cell Biology 163, 583-595.

$95110.1083 / \mathrm{jcb} .200305010$.

952 
bioRxiv preprint doi: https://doi.org/10.1101/2021.02.28.433274; this version posted September 3, 2021. The copyright holder for this preprint (which was not certified by peer review) is the author/funder. All rights reserved. No reuse allowed without permission.

953 


\section{Figure legends}

956 Figure 1. Type 1 collagen induces elongation of MCF10A anlage via cell 957 proliferation.

958 (A) Cartoon: transplantation of MCF10A cell aggregates from Matrigel ${ }^{\mathrm{TM}}$ into 959 collagen gel. Single isolated cells were cultured on Matrigel ${ }^{\mathrm{TM}}$ and overlayed with 960 Matrigel $^{\mathrm{TM}}$-containing medium. Matrige ${ }^{\mathrm{TM}}$ was washed out after 10 days and the 961 aggregates re-embedded into type-1 collagen gel.

962 (B) Time lapse images of MCF10A aggregates after transfer into a collagen gel. Red 963 arrowheads: elongation from aggregates.

964 (C) Fluorescence image of an elongated aggregate cultured for 8 days after transfer 965 into collagen gel and stained with anti-Ki67 antibody (green), phalloidin (magenta) 966 and DAPI (blue). Blue parenthesis: non-elongating area, red parenthesis: elongating 967 area.

968 (D) Percentage of Ki67 positive cells in elongating and non-elongating areas of the 969 elongated aggregates. ( $n=15$ aggregates).

970 (E) Time lapse images of elongating aggregates expressing NLS-mCherry. Yellow 971 arrowheads: dividing nucleus.

972 (F) Frequency of cell division in elongating and non-elongating regions of aggregates. 973 ( $n=12$ aggregates).

974 (G) Percentage of Ki67-positive cells in aggregates cultured for 0-3 days. ( $\mathrm{n}=102$ 975 aggregates).

976 (H) Symmetry ratio of aggregates cultured for 0-3 days. ( $n=102$ aggregates).

977 (I) Percentage of Ki67 positive cells in elongating area and non-elongating areas of 978 aggregates which broke symmetry (defined as symmetry ratio $>1.5$ ) in the first 3 979 days of culture. ( $\mathrm{n}=18$ aggregates).

980 (J) MCF10A aggregates co-stained with anti-Ki67 antibody (green) and DAPI (blue). 981 Aggregates were cultured for 0 day (i) or 8 days (ii-iv) after treatment with vehicle (ii), 982 mitomycin C (iii) or aphidicolin (iv).

983 (K) Percentage of Ki67 positive cells in aggregates cultured for 1 day or 8 days with 984 or without mitomycin $\mathrm{C}$ or aphidicolin. ( $\mathrm{n}=63$ aggregates).

985 (L) Symmetry ratio of aggregates cultured for 1 day or 8 days with or without 986 mitomycin $\mathrm{C}$ or aphidicolin. ( $\mathrm{n}=237$ aggregates).

987 (M) Effect of delayed inhibition of proliferation on aggregate elongation. Aggregates 988 were cultured for 3 days before treatment with mitomycin $\mathrm{C}$ or aphidicolin. Data are 989 fold change of elongation in control and drug-treated cultures ( $n=57$ aggregates).

990 All data are means $\pm \mathrm{SEM},{ }^{*} \mathrm{P}<0.05$, ${ }^{* *} \mathrm{P}<0.01,{ }^{* * *} \mathrm{P}<0.001$. Data in $(\mathrm{D}, \mathrm{F}, \mathrm{I})$ were 991 analyzed by unpaired Student's $t$-test. Data in ( $\mathrm{G}, \mathrm{H}, \mathrm{K}-\mathrm{M})$ were analyzed with one992 way ANOVA Tukey's multiple comparisons test.

993 The following figure supplements are available for figure 1:

994 Figure supplement 1. 3D morphology of MCF10A cells.

995 Figure supplement 2. Cell division and motility during aggregate elongation.

Figure 2. Mammary cell aggregates polarize the ECM as they elongate. 
998 (A) Fluorescent image of collagen fiber alignment and elongated aggregates

999 expressing the cell membrane marker GFP-HRasC20. Collagen fibers were labeled 1000 with mCherry-CNA35 peptide (magenta).

1001 (B) Cartoon of symmetry ratio of aggregates (i) and gel coherency (ii).

1002 (C) Scatter plot of aggregate symmetry ratio and collagen fiber coherency. $(n=75$ 1003 aggregates).

1004 (D) Regional analysis of collagen coherency around aggregates. Cartoon illustrates 1005 the approach: for elongating aggregates coherency was measured in regions of 1006 interest (ROIs) placed both at the tips of elongations and proximate to their non1007 rounded areas. For rounded aggregates, ROls were placed orthogonally. Regional 1008 differences in coherency were measured as the fold difference, measured around 1009

1010 rounded aggregates ( $n=19$ aggregates) and elongated aggregates $(n=57$

1011 aggregates). Elongated aggregates were defined as symmetry ratio $>1.5$. Double headed arrow: elongating axis.

1012 (E) Fold difference of collagen fiber coherency around aggregates measured at early 1013 stages of elongation (first 3 days of culture) subdivided based on symmetry ratio 1014 ( $\mathrm{n}=98$ aggregates).

1015 (F) Fluorescent images of collagen fibers labeled with mCherry-CNA35 (magenta) 1016 and aggregates expressing GFP-HRasC20 cultured for 8 days with mitomycin $\mathrm{C}$ or 1017 aphidicolin.

1018 (G) Coherency of collagen fibers surrounding the aggregates treated with mitomycin $1019 \mathrm{C}$ and aphidicolin. ( $\mathrm{n}=40$ aggregates).

$1020(\mathrm{H})$ Fluorescent images of collagen fiber alignment with aggregates expressing GFP1021 HRasC20 treated with blebbistatin or Y-27632 for 8 days. Collagen fibrils were 1022 labeled with CNA35-mCherry (magenta).

1023 (I) Symmetry ratio of aggregates cultured for 8 days with or without blebbistatin or $Y$ 1024 27632. ( $n=88$ aggregates).

1025 (J) Distribution of collagen fiber orientation surrounding elongated aggregates ( $\mathrm{n}=$ 102622 aggregates) or non-elongated aggregates ( $n=13$ aggregates).

1027 (K) Difference between elongation axis of aggregates and average angle of collagen 1028 fibers in non-elongating area or elongating area. ( $n=57$ aggregates).

1029 All data are means \pm SEM, ns, not significant, ${ }^{*} \mathrm{P}<0.05,{ }^{* *} \mathrm{P}<0.01,{ }^{* *} \mathrm{P}<0.001$. Data 1030 in

1031 (D, E, G, I) were analyzed with one-way ANOVA Tukey's multiple comparisons test. 1032 Data in (K) were analyzed by unpaired Student's $t$-test.

1033 The following figure supplements are available for figure 2:

1034 Figure supplement 1. Immunofluorescent staining of ECM proteins in the 1035 aggregates.

1036 Figure supplement 2. Effect of Rac1 GEF inhibitor on MCF10A aggregate 1037 elongation.

1038

1039

Figure 3. Collagen polarization induces mammary aggregate elongation. 
1040 (A) Second harmonic generation (SHG) images of collagen fibers in the gel with or 1041 without stretching and incubated for 7 days after re-embedding in gel. Double head 1042 arrow: stretching axis.

1043 (B) Coherency of collagen fiber in the gel with or without stretching. ( $n=24$ positions 1044 in multiple gels).

1045 (C) Distribution of collagen fiber orientation in the gel with or without stretching. $0^{\circ}$ is 1046 defined as the axis of stretch. ( $N=3$ independent experiments).

1047 (D) SHG images of collagen fiber floated for 7 days with or without after stretching. 1048 Double head arrow: stretching axis.

1049 (E) Coherency of collagen fiber in 7 days floated gel with or without stretching. $(n=$ 105046 positions in multiple gels).

1051 (F) Distribution of collagen fiber orientation in gels that had been allowed to float (7 1052 days) with or without prior stretching. ( $N=3$ independent experiments).

1053 (G) Time lapse images of aggregates embedded in stretched gel. Double head 1054 arrow: stretching axis.

1055 (H) Initiation time of aggregate elongation in the gel with or without stretching. (1056 Stretch: $\mathrm{n}=25$ aggregates, +Stretch: $\mathrm{n}=71$ aggregates).

1057 (I) Symmetry ratio of aggregates in the early phase of culture (0-3 days) with or 1058 without stretching. (-Stretch, day $0: n=12$, day $1: n=12$, day $2: n=24$, day $3: n=$ $105924,+$ Stretch, day $0: n=21$, day $1: n=32$, day $2: n=17$, day $3: n=22)$.

1060 (J) Distribution of elongation axes of aggregates in the gel with or without stretching. 1061 (- Stretch: $\mathrm{n}=112$ aggregates, +Stretch: $\mathrm{n}=115$ aggregates).

1062 (K) Difference between elongating axis of aggregates and average angle of collagen 1063 fibers in the gel with or without stretching. (- Stretch: $n=21$ aggregates, + Stretch: $n$ $1064=102$ aggregates).

1065 (L) Fluorescence image of aggregates cultured for 5 days after gel stretching and co1066 stained with phalloidin (green) and DAPI (blue) in stretched gel. Collagen fibrils were 1067 labeled with CNA35-mCherry (magenta).

1068 (M) Coherency of collagen fibers surrounding elongated aggregates in the gel with or 1069 without stretching. (- Stretch: $n=21$ aggregates, +Stretch: $n=102$ aggregates).

1070 All data are means \pm SEM; ns, not significant, ${ }^{* *} \mathrm{P}<0.01,{ }^{* *} \mathrm{P}<0.001$. Data in $(B, E, H$, $1071 \mathrm{~K}, \mathrm{M})$ were analyzed by unpaired Student's $t$-test. Data in (I) was analyzed with one1072 way ANOVA Tukey's multiple comparisons test.

1073 The following figure supplements are available for Figure 3:

1074 Figure supplement 1. External gel stretching aligns collagen fiber.

1075 Figure supplement 2. MCF10A aggregates elongate along the gel stretching axis.

Figure 4. Collagen polarization must be sustained to stimulate mammary aggregate elongation.

1079 (A) Population of elongated aggregates in the re-embedded or floated gel. ( $\mathrm{N}=3$ 1080 independent experiments).

1081 (B) The length of elongated aggregates in the re-embedded or floated gel. ( $N=3$ 1082 independent experiments). 
1083 (C) Coherency of collagen fibers surrounding the aggregates in gels that were re1084 embedded after stretch or floated for 7 days after stretching. ( $n=68$ aggregates).

1085 (D) Cartoon of aggregate from stretched gel into normal collagen gel. Aggregates 1086 were isolated from stretched gel by collagenase and re-embedded in naïve gel.

1087 (E) Initiation time for aggregate elongation in non-stretched control gels, stretched 1088 gels that had been re-embedded to preserve collagen polarization (embed) and after 1089 cells were extracted and transferred into non-stretched gels (transfer) $(n=83$ 1090 aggregates).

1091 All data are means \pm SEM; ns, not significant, ${ }^{* *} \mathrm{P}<0.01,{ }^{* *} \mathrm{P}<0.001$. Data in $(\mathrm{A}-\mathrm{C})$ 1092 were analyzed by unpaired Student's $t$-test. Data in (E) was analyzed with one-way

Figure 5. Collagen polarization induces cell proliferation for aggregate elongation.

1097 (A) Time course of cell proliferation within aggregates in control gels (- stretch) or 1098 after stretching (+ stretch). Data are percentage of cells that were Ki67 positive ( $\mathrm{n}=$ 206 aggregates).

$1100(\mathrm{~B}, \mathrm{C})$ Length $(\mathrm{B})$ and $(\mathrm{C})$ symmetry ratio of aggregates in stretched gel incubated with mitomycin $C$ or aphidicolin for 8 days. ( $n=214$ aggregates)

(D) Fluorescence images of elongated aggregates cultured for 7 days in the reembedded or floated gel after stretching. Aggregates were co-stained with anti-Ki67 antibody (green), Phalloidin (red) and DAPI (blue). Collagen fibers were labeled with mCherry-CNA35 (magenta).

1106 (E) Percentage of Ki67 positive cells in the aggregates cultured for 7 days in the reembedded or floated gel after stretching. ( $n=56$ aggregates).

1108 (F) Percentage of Ki67 positive cells in the elongating area of aggregates in the reembedded or floated gel. ( $n=47$ aggregates).

All data are means \pm SEM; ns, not significant, ${ }^{* *} \mathrm{P}<0.01,{ }^{* *} \mathrm{P}<0.001$. Data in $(E, F)$ one-way ANOVA Tukey's multiple comparisons test.

Figure 6. Polarized collagen promotes cell proliferation via the ERK pathway.

(B) Percentage of ERK active cells in elongating areas and non-elongating areas of the aggregates. ( $n=30$ aggregates). areas of aggregates. ( $n=30$ aggregates).

(D) Fluorescent images of aggregates cultured for 7 days treated with FR180207 after gel stretching. Aggregates were co-stained with anti-Ki67 antibody (green), Phalloidin (red) and DAPI (blue). Collagen fibers were labeled with mCherry-CNA35 (magenta).

1125 (E) Percentage of Ki67-positive cells in aggregates incubated with FR180207 for 7days after stretching. ( $n=24$ aggregates). 
1127 (F) Effect of inhibiting ERK on stretch-induced aggregate elongation. Proportion of

1128 elongated aggregates in stretched gel incubated with FR180207 for 3 days and 7 1129 days. ( $\mathrm{N}=3$ independent experiments).

$1130(\mathrm{G})$ Length and $(\mathrm{H})$ symmetry ratio of elongated aggregates incubated with 1131 FR180207 for 7 days. ( $n=166$ aggregates).

1132 All data are means \pm SEM; ns, not significant, ${ }^{* \star} \mathrm{P}<0.01,{ }^{* \star *} \mathrm{P}<0.001$. Data in $(\mathrm{B}, \mathrm{C}, \mathrm{E}$, $1133-\mathrm{H}$ ) were analyzed by unpaired Student's $t$-test.

1134 The following figure supplement is available for figure 6:

1135 Figure supplement 1. ERK biosensor and YAP1 localization in MCF10A cells.

Figure 7. Integrins are necessary for polarized collagen to stimulate elongation.

(A) Schematic of potential integrin-ERK pathway that mediates the effect of collagen polarization on cell proliferation.

(B) Immunoblot of integrin $\alpha 2, \beta 1$ and ERK1/2 protein levels in MCF10A cell lysate.

(C) Fluorescent images of aggregates cultured for 7 days treated with AllB2 antibody (magenta).

(D) Percentage of Ki67 positive cells in the aggregates incubated with AllB2 antibody

(E) Proportion of elongated aggregates in stretched gel incubated with AllB2 antibody for 3 days and 7 days. ( $N=3$ independent experiments).

$(F)$ Length and $(G)$ symmetry ratio of elongated aggregates incubated with AllB2 antibody for 7 days. ( $n=134$ aggregates).

(H) Fluorescent images of aggregates expressed with ERK/KTR-mClover and incubated with IgG (control) or AllB2 antibody for 2 days after gel stretching. Aggregates were co-stained with phalloidin (red) and DAPI (blue). Collagen fibers were labeled with mCherry-CNA35 (magenta).

1155 (I) Percentage of ERK active cells in aggregates incubated with AlIB2 antibody for 2 days or 7 days in stretched gel. ( $n=78$ aggregates).

All data are means \pm SEM; ns, not significant, ${ }^{* *} \mathrm{P}<0.01,{ }^{* *} \mathrm{P}<0.001$. Data in $(\mathrm{D}-\mathrm{G}, \mathrm{I})$ were analyzed by unpaired Student's $t$-test.

Figure 8. Model of collagen polarization as a structural memory for epithelial anlage elongation.

(i) Initially isotropic epithelia anlage exert isotropic patterns of force on a nonpolarized collagen $1 \mathrm{gel}$.

1164 (ii) Initial anisotropies in force associated with symmetry-breaking of the aggregate exert strain on collagen fibrils leading to bundling and polarization. regional cell proliferation to direct further elongation of the anlage.

\section{Supplemental Figure legends}


1171 Figure 1-figure supplement 1. 3D morphology of MCF10A cells.

1172 (A) MCF10A cells cultured in Matrigel ${ }^{\mathrm{TM}}$ for 7 days (left) and 31 days (right). Day 7 1173 aggregate was co-stained with anti-E-cadherin antibody (green), anti-GM130 1174 antibody (red), phalloidin (magenta) and DAPI (blue). Day 31 acinus was co-stained 1175 with anti-Laminin V antibody (green), anti-E-cadherin antibody (red) and DAPI (blue). 1176 (B) MCF10A cells cultured in Type 1 collagen gel for 3 days (left) and 10 days (right). 1177 Day 3 MFC10A cells expressing GFP-HRasC20 were co-stained with anti-GM130 1178 antibody (red), phalloidin (magenta) and DAPI (blue). Day 10 MCF10A cells were co1179 stained with phalloidin (magenta) and DAPI (blue).

1180 (C) MCF10A aggregate cultured for 7 days after transplantation from Matrigel ${ }^{\mathrm{TM}}$ back 1181 into Matrigel ${ }^{\mathrm{TM}}$. Aggregate was co-stained with anti-Ki67 antibody (green), phalloidin 1182 (red) and DAPI (blue).

Figure 1-figure supplement 2. Cell division and motility during aggregate elongation.

1186 (A) Average angle of cell division. Principal axis of aggregate is $0^{\circ}$. $(\mathrm{n}=12$ aggregates).

1188 (B) Nuclear tracking during aggregate elongation. Tails show tracking path for each cell in the last 120 min of the movie.

(C) Speed of cell motility based on nuclear tracking in rounded and elongated aggregates treated with mitomycin $\mathrm{C}$ or aphidicolin. ( $\mathrm{n}=137$ movies).

(D) Cell speed, (E) Track straightness and (F) track displacement angle in elongating aggregates. ( $\mathrm{n}=18$ movies).

$1194(\mathrm{G})$ Track straightness and $(\mathrm{H})$ track displacement angle of cells in aggregates treated with mitomycin $\mathrm{C}$ or aphidicolin ( $\mathrm{n}=60$ movies).

1196 All data are means \pm SEM; ns, not significant, ${ }^{*} P<0.05,{ }^{* *} P<0.001$. Data in (D-F) were analyzed by unpaired Student's t-test. Data in $(\mathrm{C}, \mathrm{G}, \mathrm{H})$ were analyzed with one-way ANOVA Tukey's multiple comparisons test.

Figure 2-figure supplement 1. Immunofluorescent staining of ECM proteins in the aggregates.

(A) MCF10A aggregates transferred from Matrige $^{\mathrm{TM}}$ into collagen gel at day 0 (left) and at day 4 (right). Day 0 aggregate was co-stained with anti-Laminin $\mathrm{V}$ antibody (green), anti-E-cadherin antibody (red), and DAPI (blue). Day 4 aggregate was costained with anti-Laminin V antibody (green), anti-E-cadherin antibody (red), phalloidin (magenta), and DAPI (blue). *: Y-Z slice image of yellow line. and at day 7 (right). Aggregates were co-stained with anti-fibronectin antibody (green) and DAPI (blue). 
1213 (A) Fluorescent images of collagen fiber alignment with aggregates expressing GFP1214 HRasC20 treated with NSC23766 for 8 days. Collagen fibers were labeled with 1215 mCherry-CNA35 (magenta).

1216 (B) Speed of cell movement based on nuclear tracking in rounded and elongated 1217 aggregates treated with NSC23766. ( $n=82$ movies)

1218 (C) Effect of delayed inhibition of proliferation on aggregate elongation. Aggregates were cultured for 3 days before treatment with NSC23766. Data are fold change of elongation in control and drug-treated cultures ( $n=40$ aggregates).

1221

1222 All data are means \pm SEM; ns, not significant, ${ }^{*} P<0.05,{ }^{* *} P<0.01$. Data in $(B, C)$ were analyzed with one-way ANOVA Tukey's multiple comparisons test. Data in (D) was analyzed by unpaired Student's $t$-test.

Figure 3-figure supplement 1. External gel stretching aligns collagen fiber.

1226

(A) PDMS gel frame and stretching device.

1227

(B) Schematic image of stretching experiment. Gel was stretched for 4 hours and then re-embedded in gel or floated in culture medium for 7 days.

1229

1230

(C) Second harmonic generation microscopy images of collagen gel with or without stretch. Double head arrow: stretching axis.

1231

(D) Coherency of collagen fibers in gel and (E) distribution of fiber orientation. ( $N=4$

1232 independent experiments)

All data are means $\pm \mathrm{SEM} ;{ }^{* *} \mathrm{P}<0.01,{ }^{* * *} \mathrm{P}<0.001$. Data was analyzed by unpaired

Student's $t$-test.

1235

Figure 3-figure supplement 2. MCF10A aggregates elongate along the gel stretching axis.

(A) MCF10A aggregates in the gel after 4 hours stretching. Aggregate was costained with Phalloidin (green) and DAPI (blue). Collagen fibers were labeled with mCherry-CNA35 (magenta).

(B) Symmetry ratio of aggregates with or without gel stretching for 4 hours ( $n=274$ aggregates).

1243 (C) Schematic image of measurement of elongation angle.

1244 (D) The average angle of elongated aggregates in non-stretched gel and stretched 1245 gel. ( $n=227$ aggregates).

1246 (E) MCF10A aggregates cultured for 1 day in non-stretched and stretched gels. 1247 Aggregates were co-stained with anti-fibronectin antibody (green) and DAPI (blue). 1248 Collagen fibrils were labeled with CNA35-mCherry (red).

1249 All data are means \pm SEM; ns, not significant, ${ }^{* *} \mathrm{P}<0.001$. Data were analyzed by 1250 unpaired Student's $t$-test.

Figure 6-figure supplement 1. ERK biosensor and YAP1 localization in MCF10A cells.

1254 (A) Time lapse images of MCF10A monolayer cells that express ERK/KTR-mClover 1255 biosensor treated with ERK inhibitor (FR180204). 
1256 (B) YAP1 localization in rounded MCF10A aggregate cultured for 1 day after transfer 1257 into collagen gel.

1258 (C) YAP1 localization in elongated MCF10A aggregate cultured for 8 days after 1259 transfer into collagen gel.

1260 Aggregates in (B,C) were co-stained with anti-YAP1 antibody (green), phalloidin 1261 (magenta) and DAPI (blue).

1262

1263

1264

1265

1266

1267

1268

1269

Video 1. Time lapse images of MCF10A aggregates after transferred into collagen gel.

Images were taken every 10 minutes.

1270

Video 2. Time lapse images of aggregates embedded in stretched gel.

Images were taken every 1 hour. 
Katsuno-Kambe et al.

Figure 1

A Matrigel overlay

\section{B 00:00}

$23: 10$

$43: 10$

bioRxiv preprint doi: https://doi.org/10.1101/2021.02.28.433274; this version posted September 3, 2021. The copyright holder for this preprint 10 days
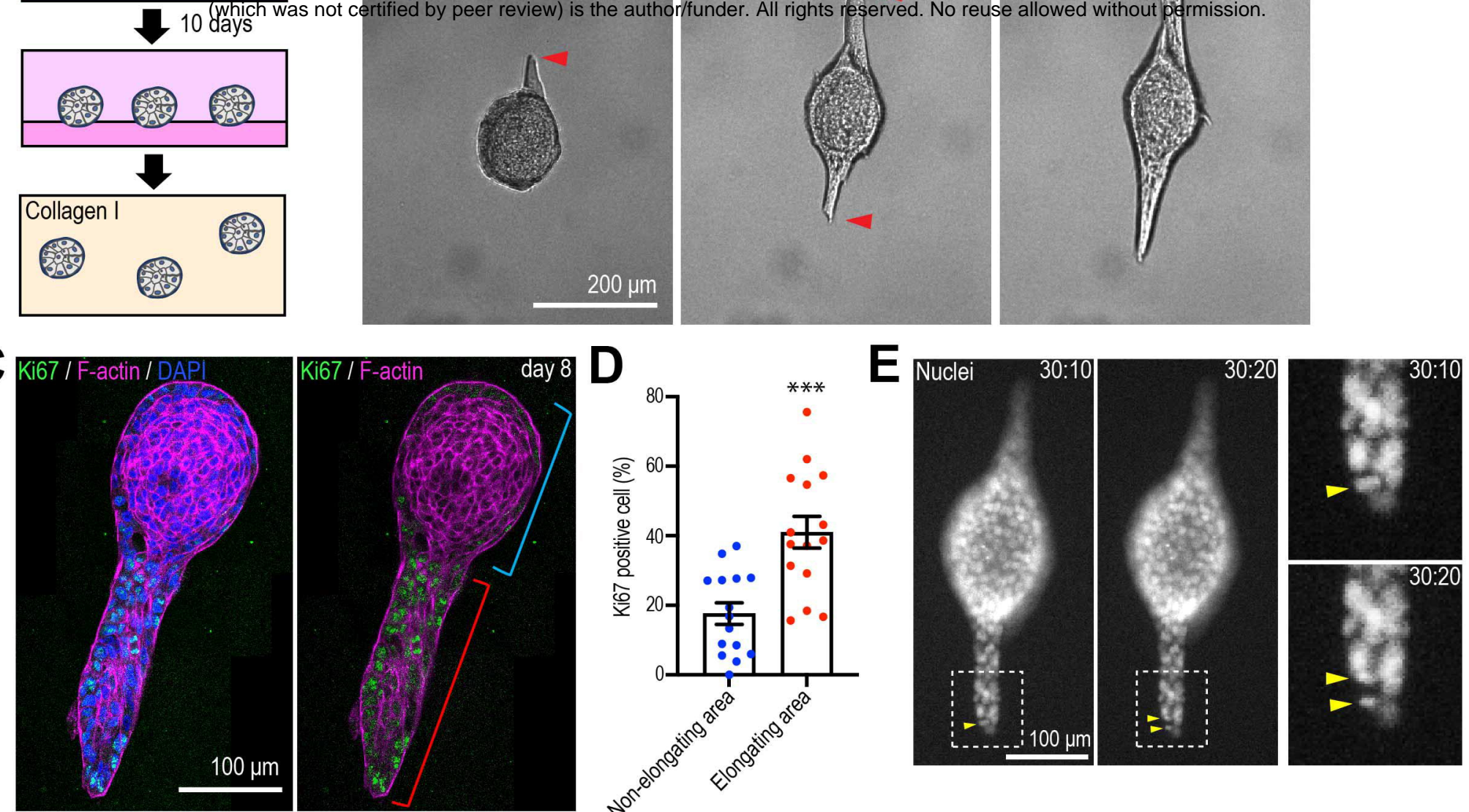

$\mathbf{F}$

G

H
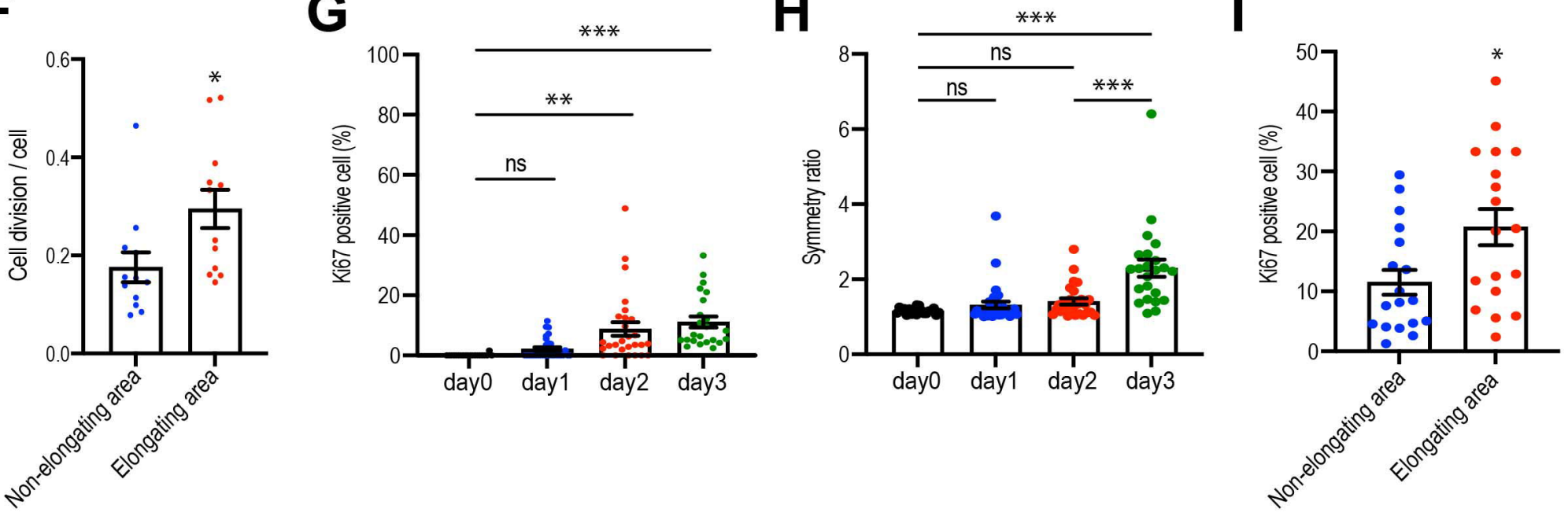
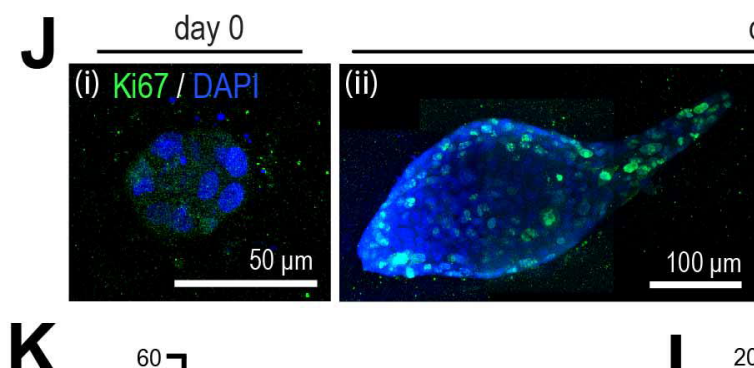

day 8
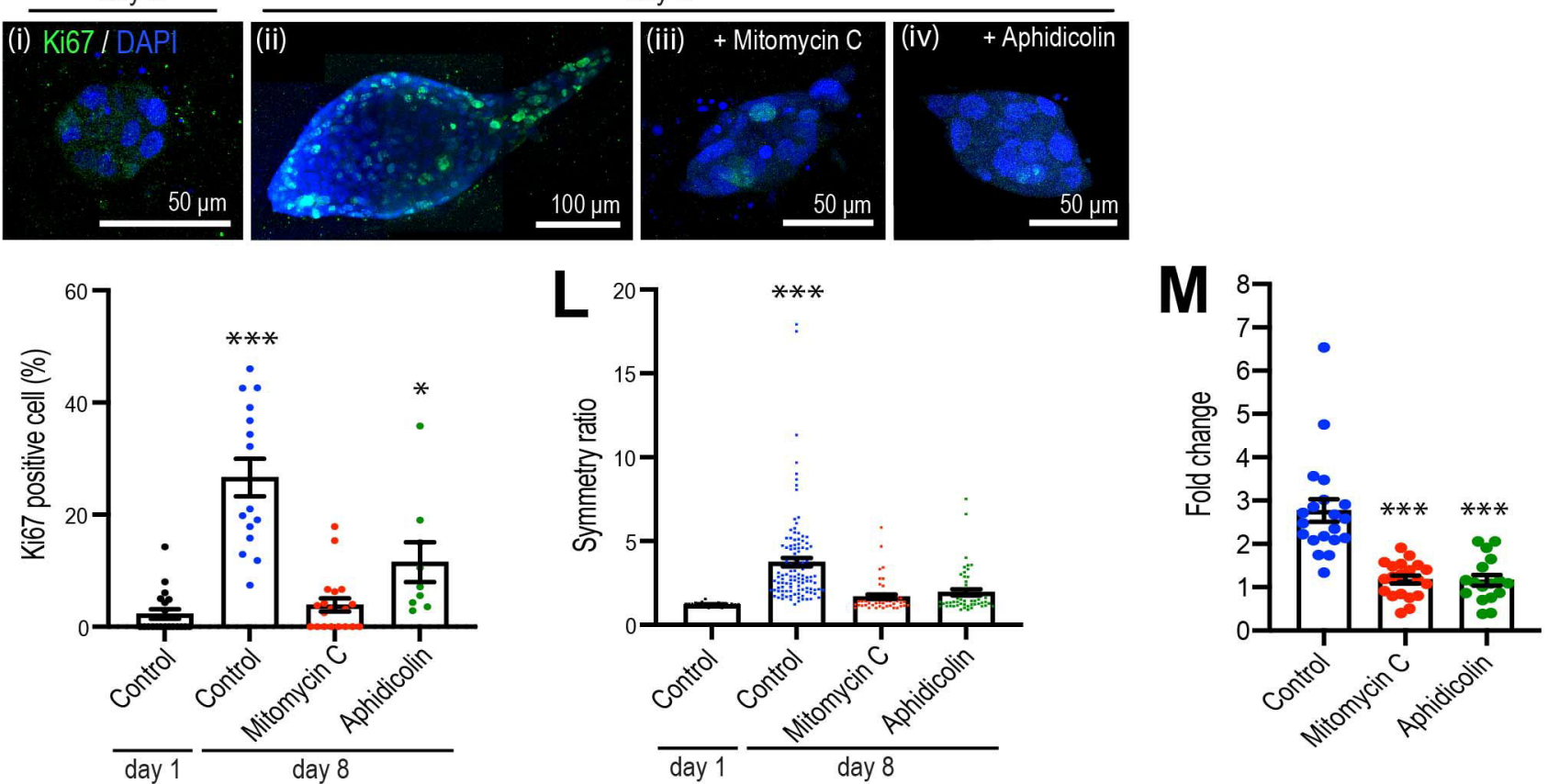

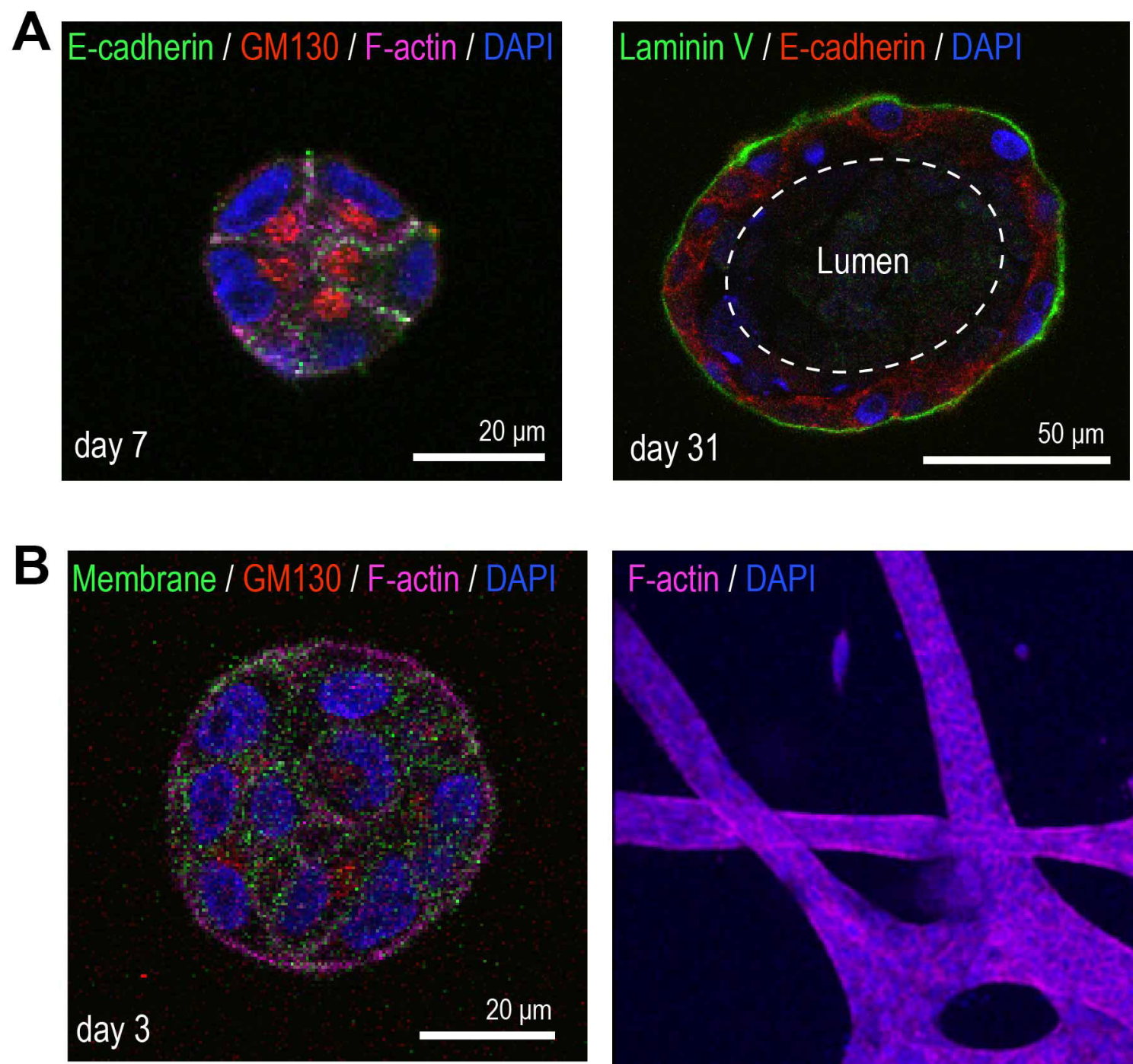

C Ki67 / F-actin / DAPI ' Matrigel 


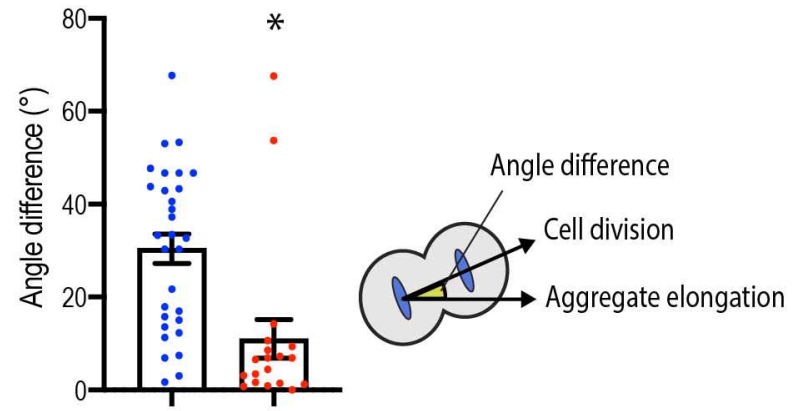

B
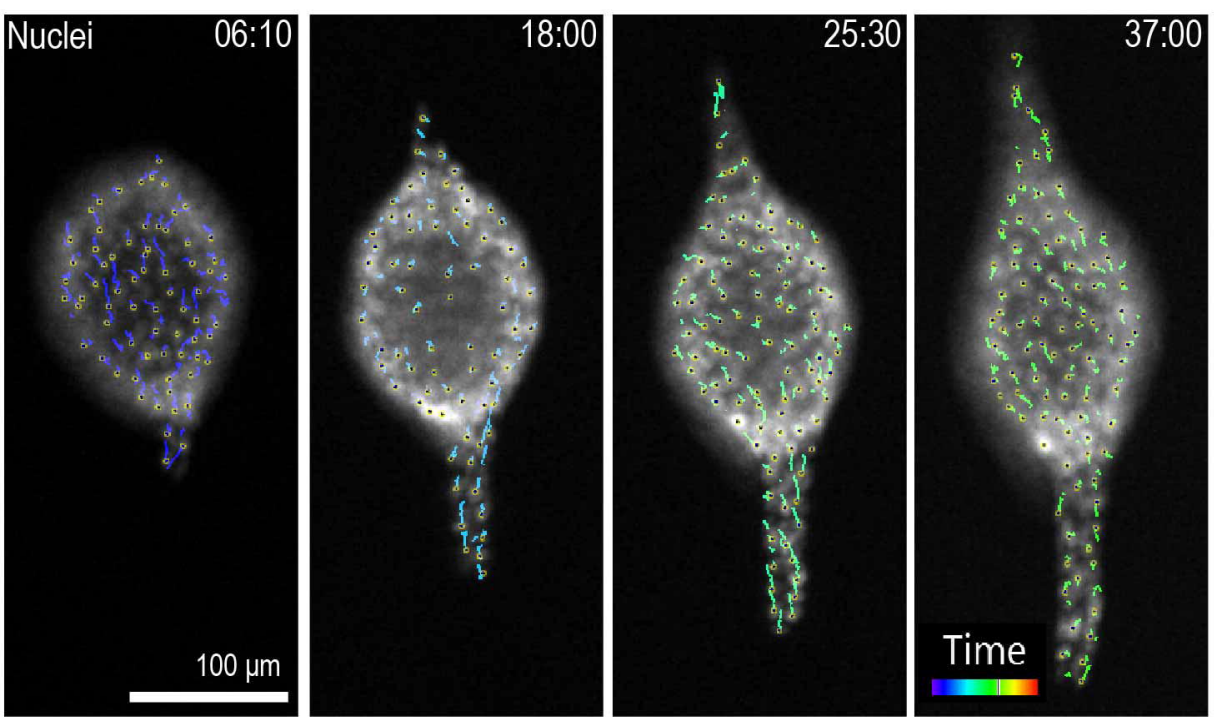

C
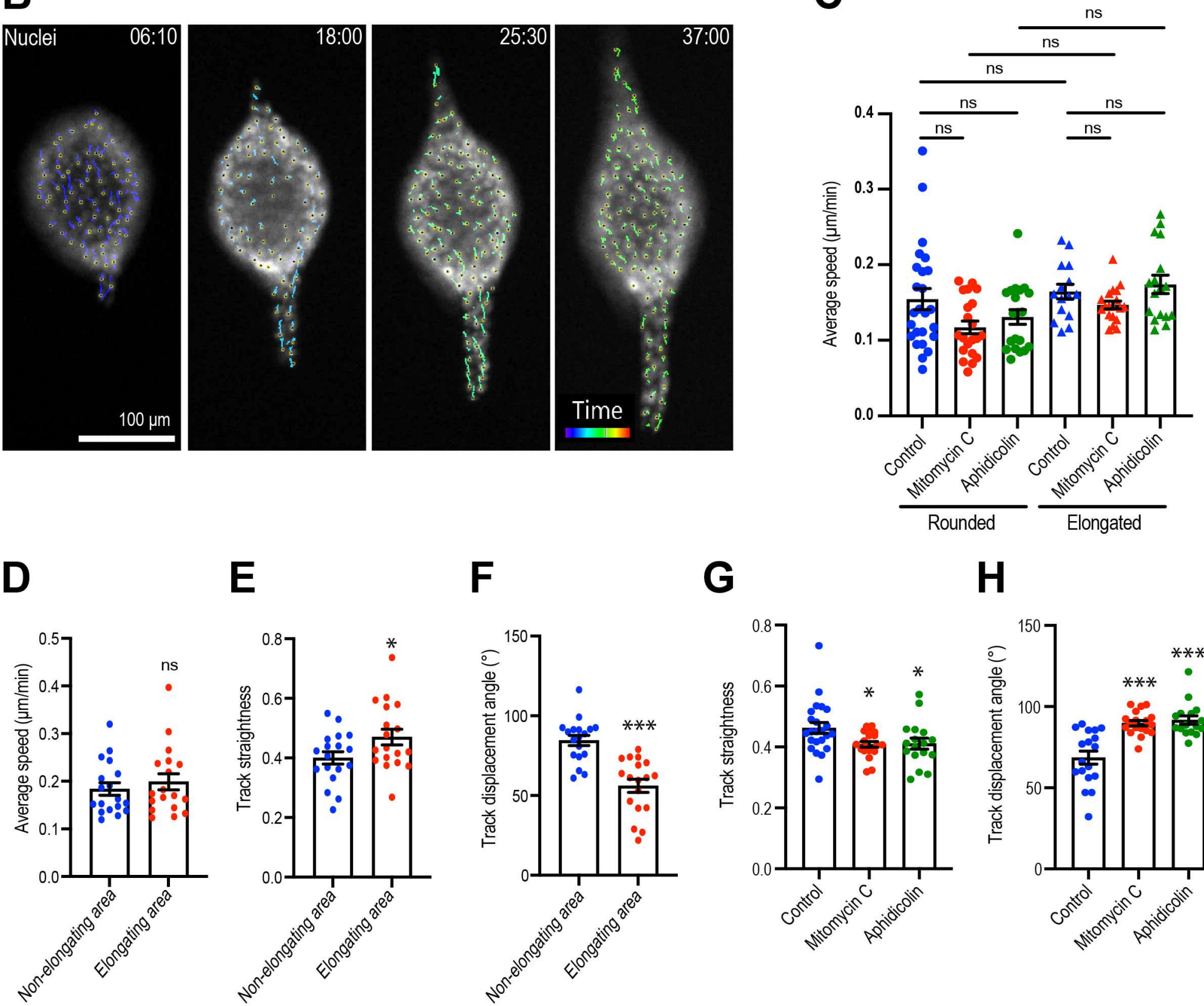

G
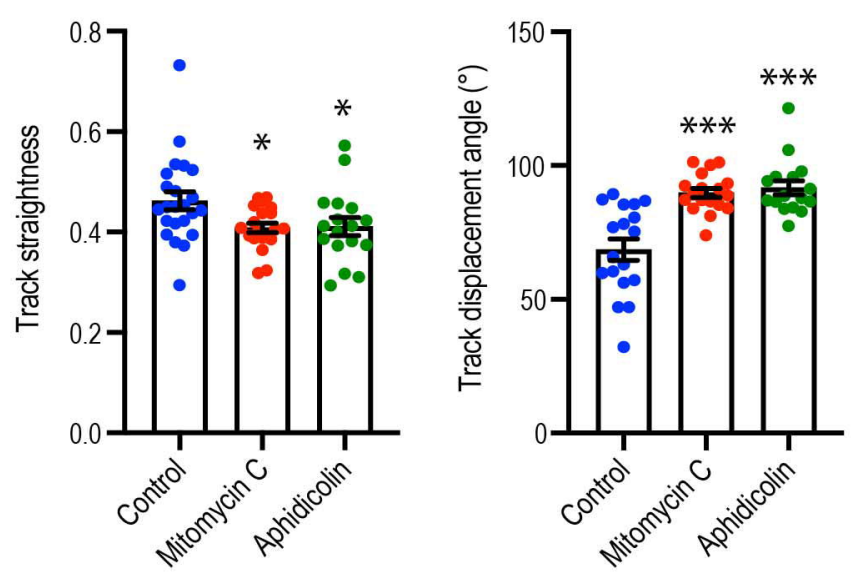
Figure 2

A bioRxiv preprint doi: https://doi.org/18101/2021.02.28.433274; this version posted Septemb Pr-2021. The copyright holder for this preprint (which was not certified b, peer review) is the author/funder. All rights reserved. Neleuse allowed without permission.

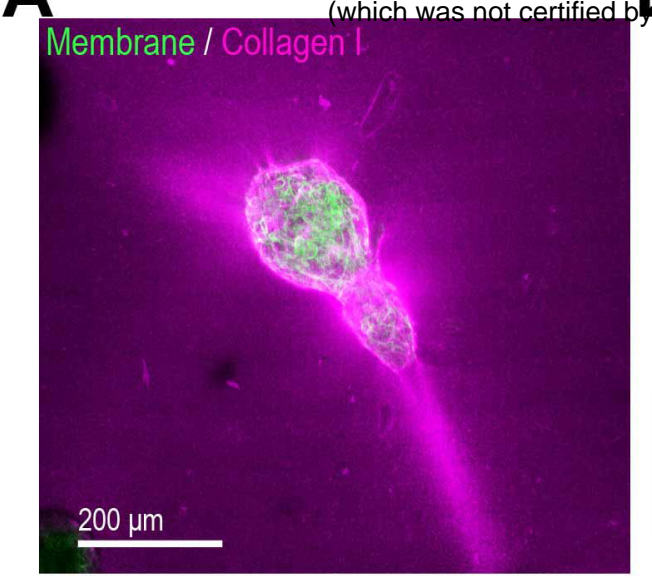

D

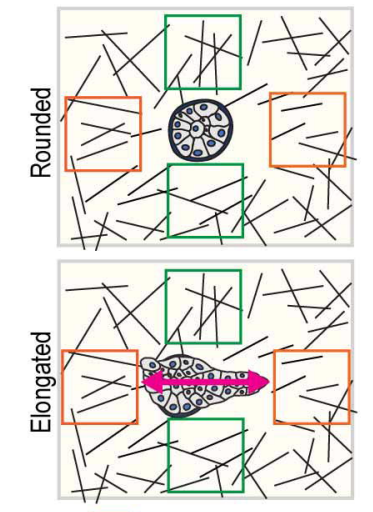

$\square$ Elongating area

$\square$ Non-elongating area

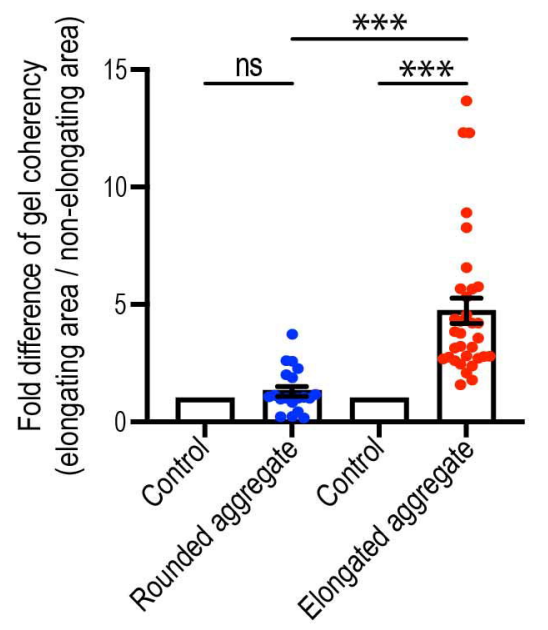

Membrane / Collagen I + Mitomycin C

(ii)

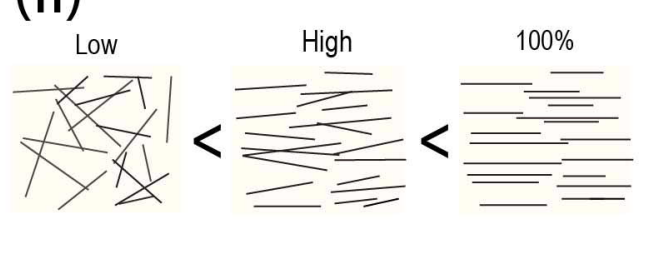

E

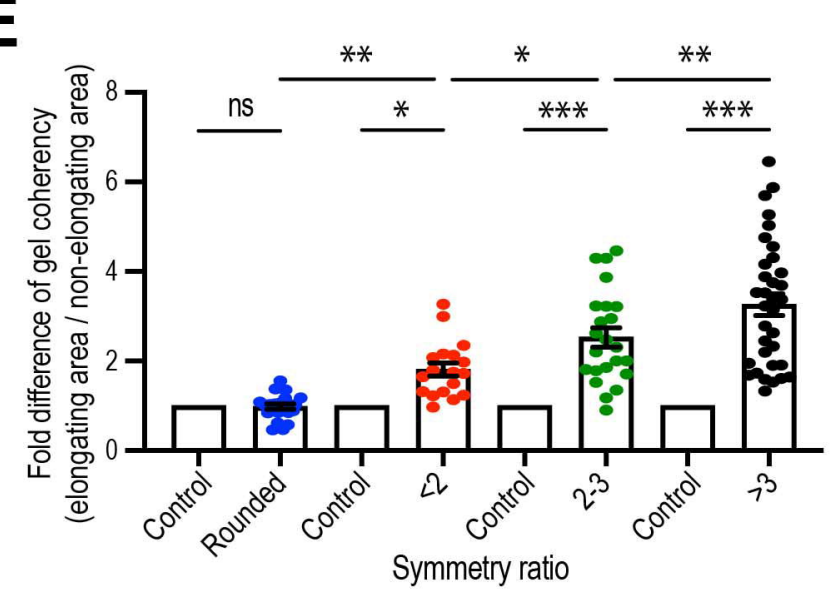

G

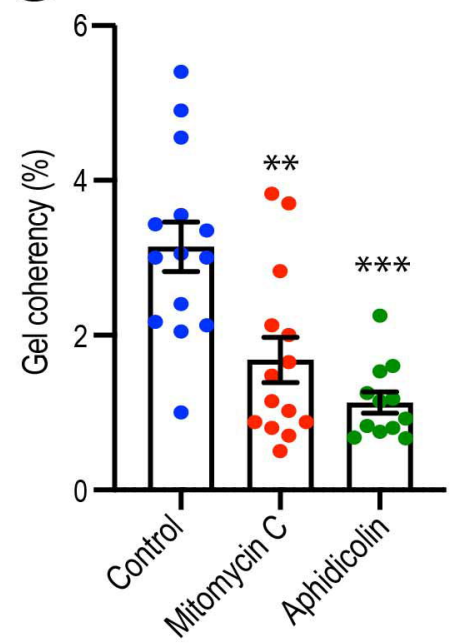

ป
Collagen fibril orientation

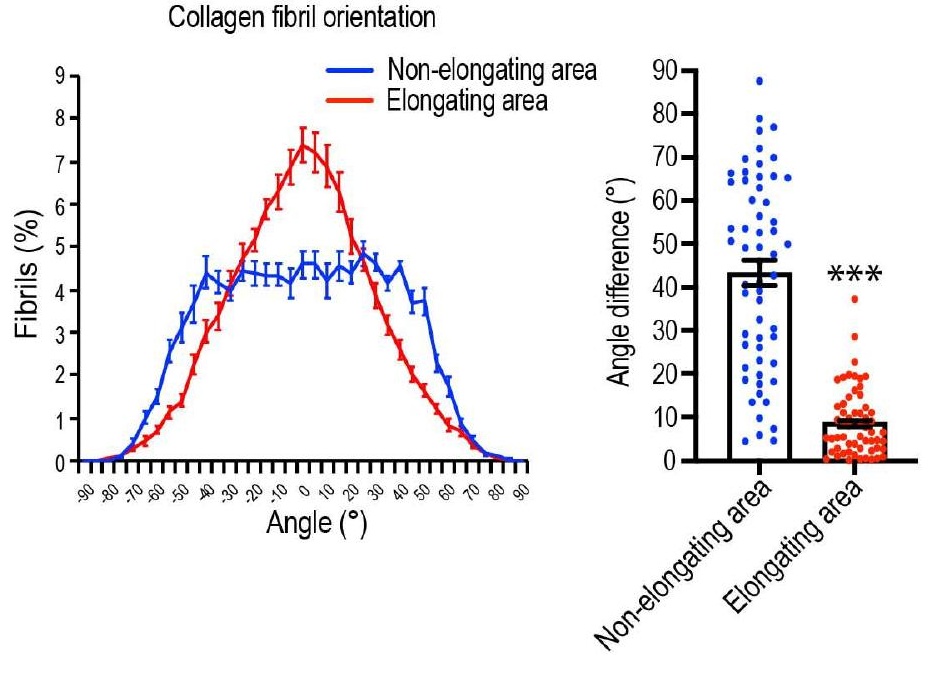

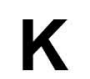

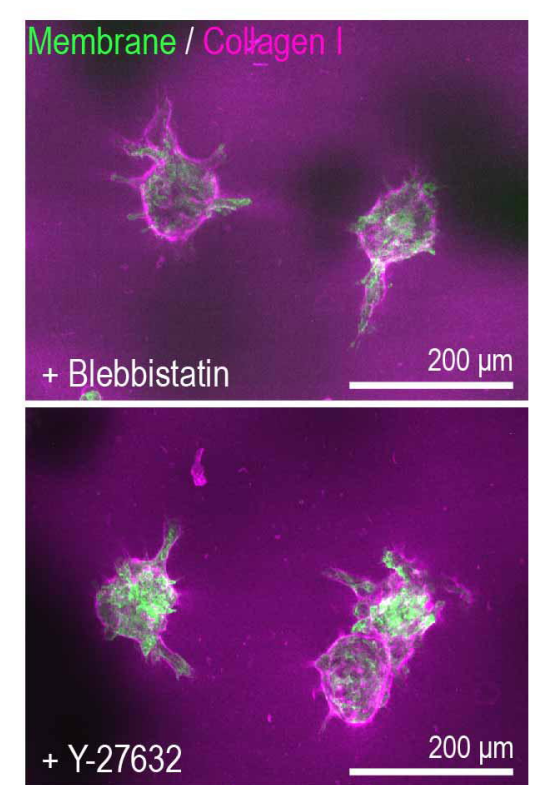

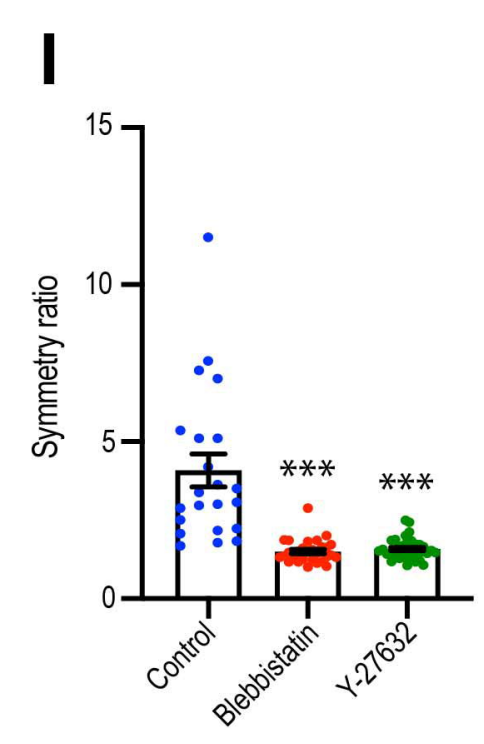

+ Aphidicolin

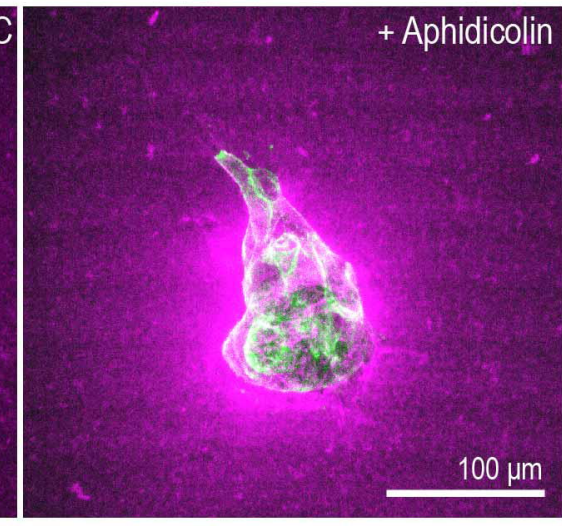




\section{A Laminin V/E-cadherin / DAPI}
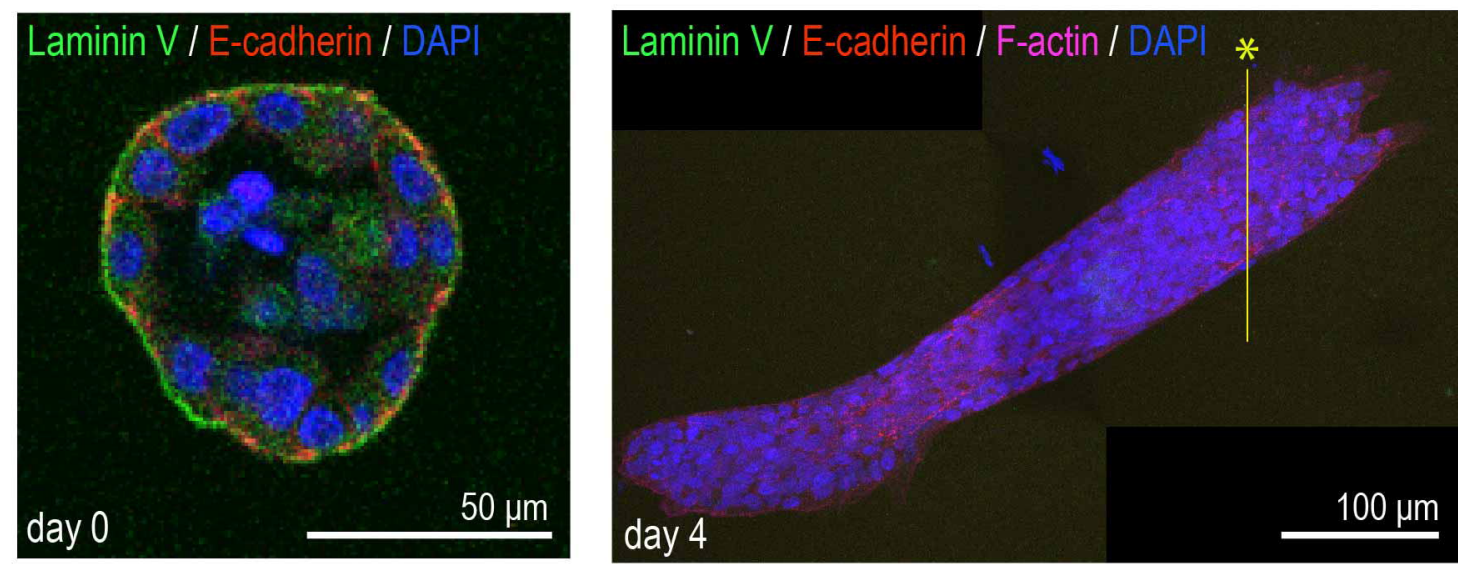

B Fibronectin / DAP

day 1

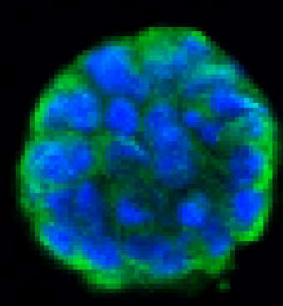

$50 \mu \mathrm{m}$

Fibronectin / DAPI

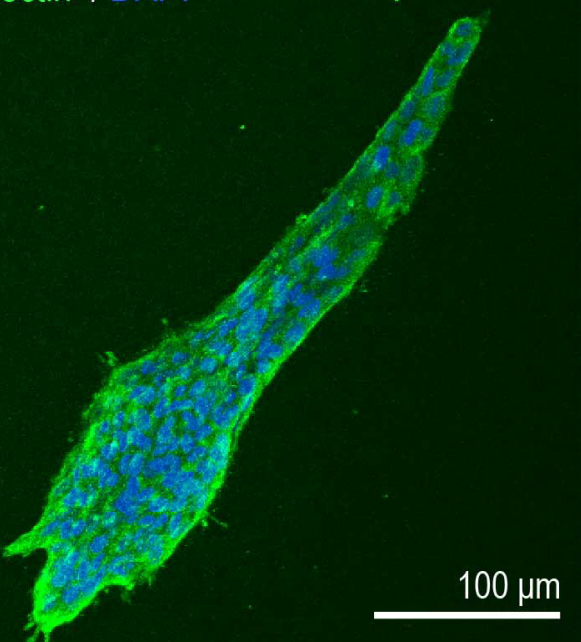


B

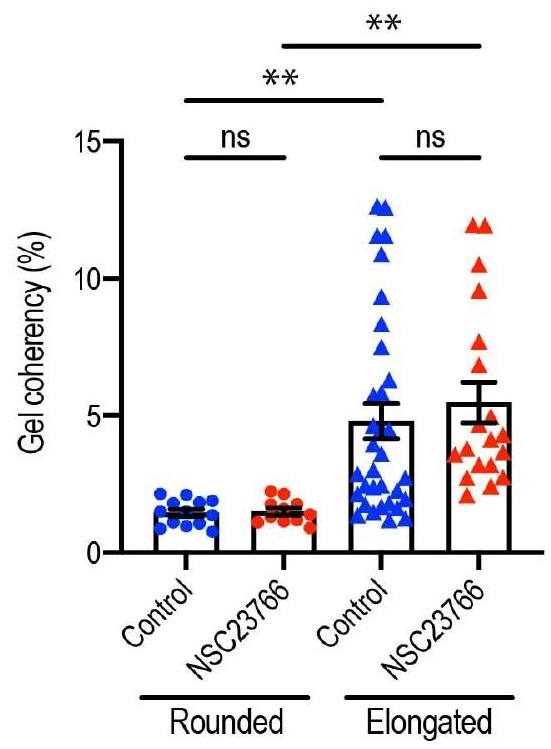

C

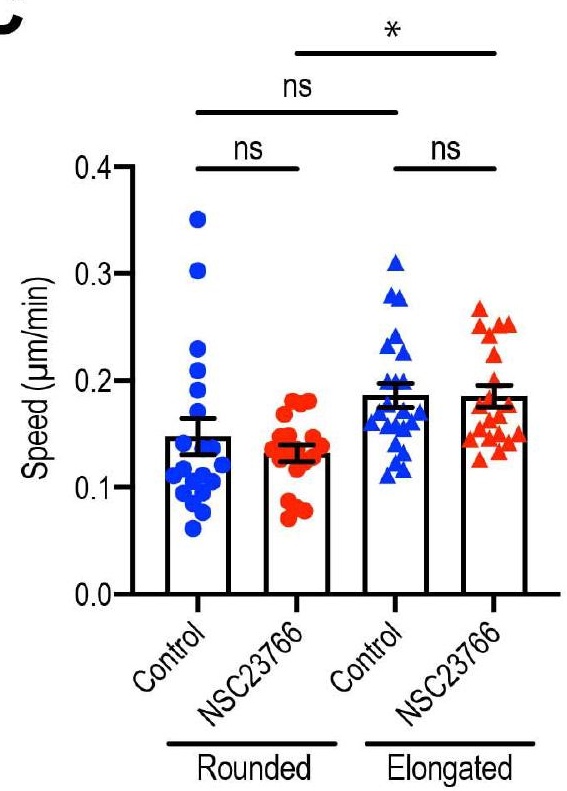

D

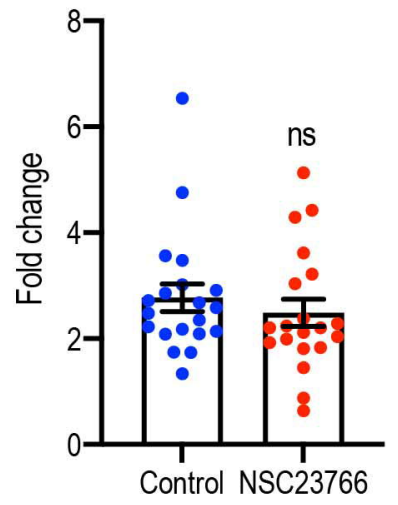




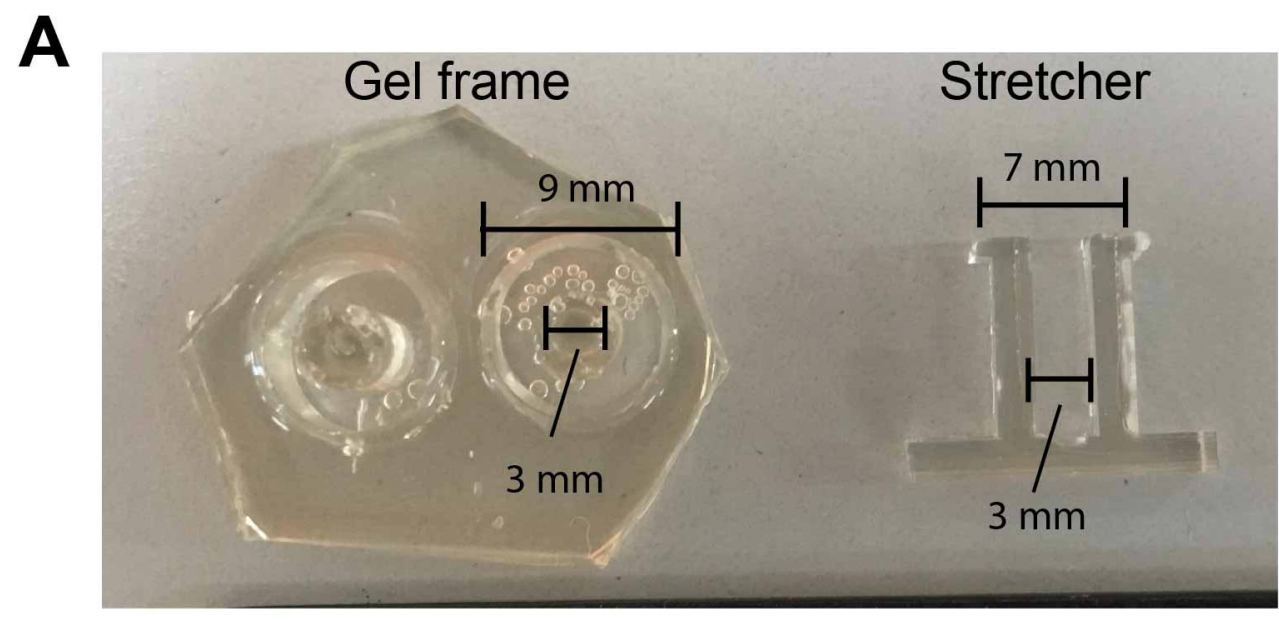

B
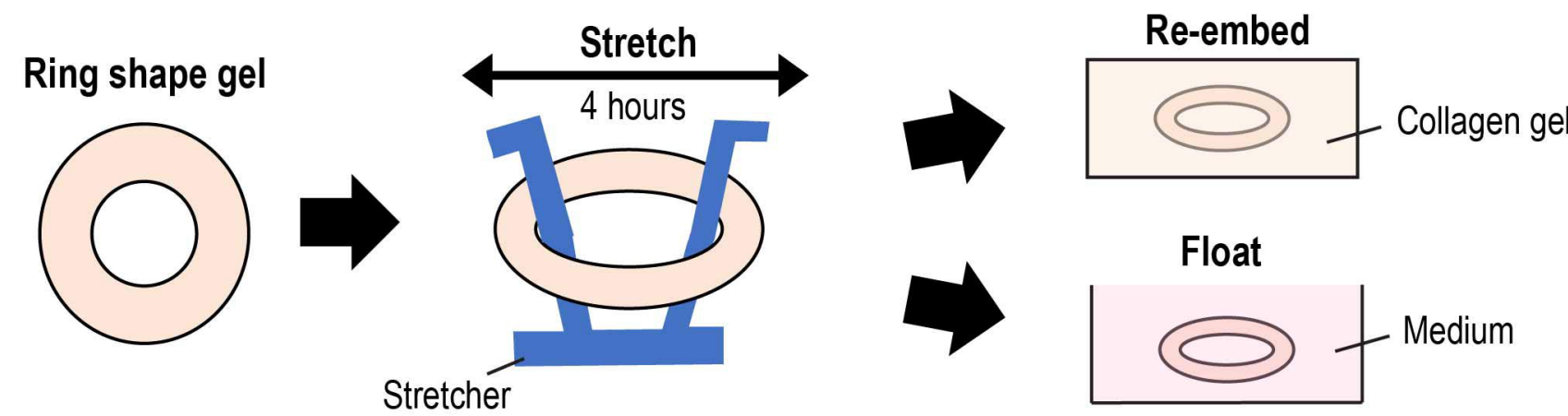

c

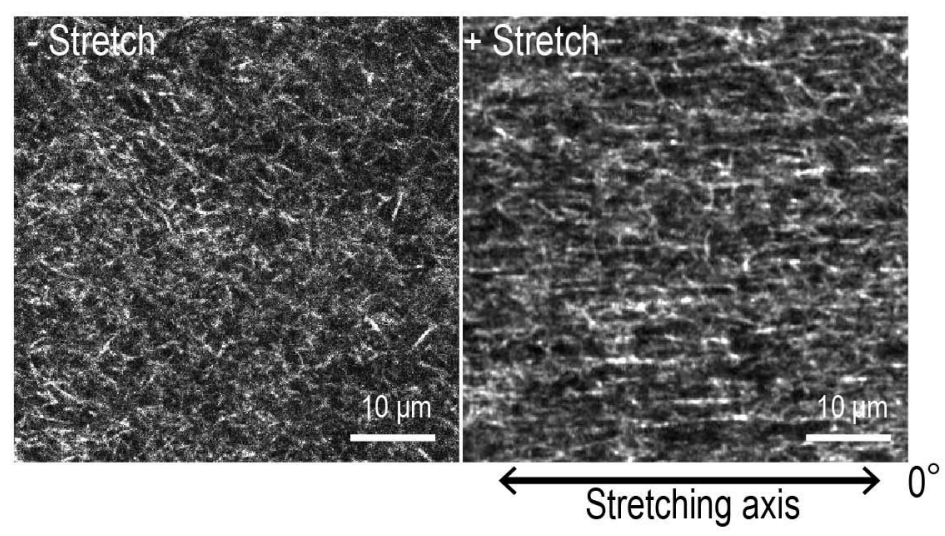

D

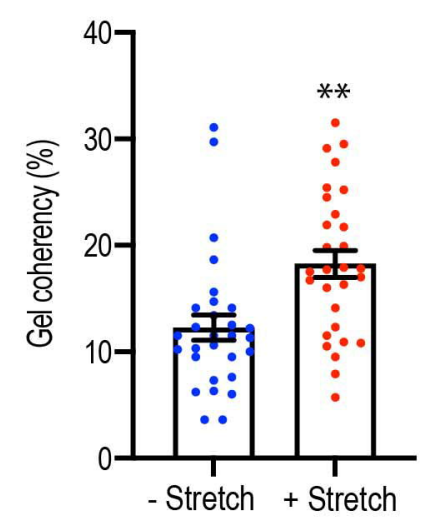

E

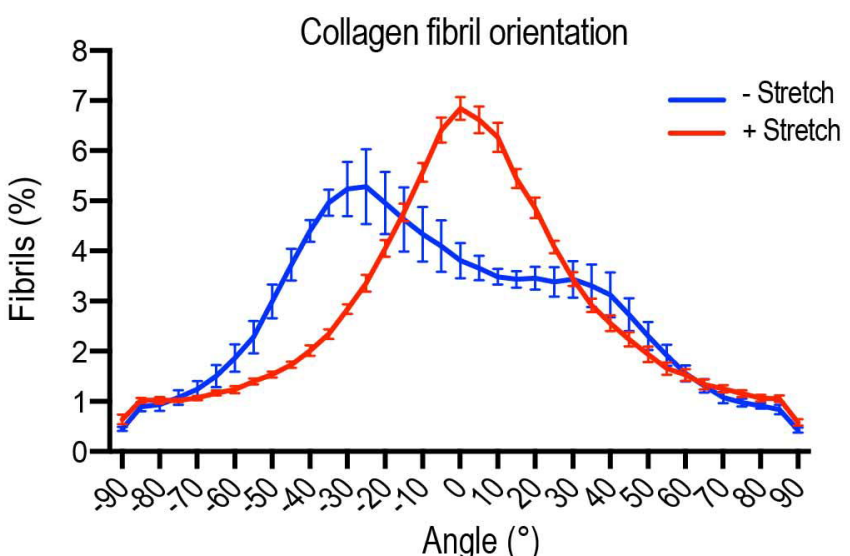


A

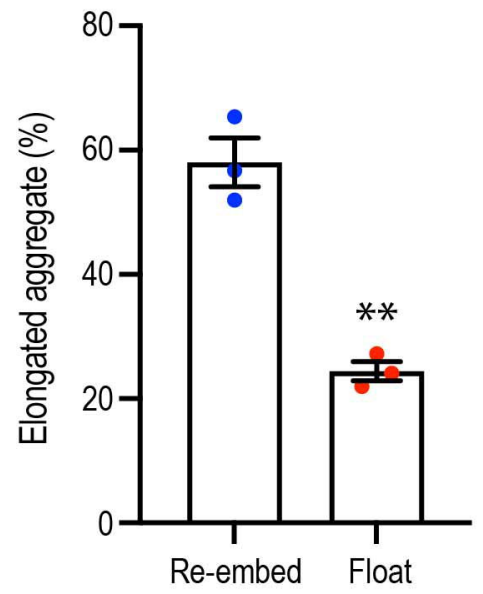

D

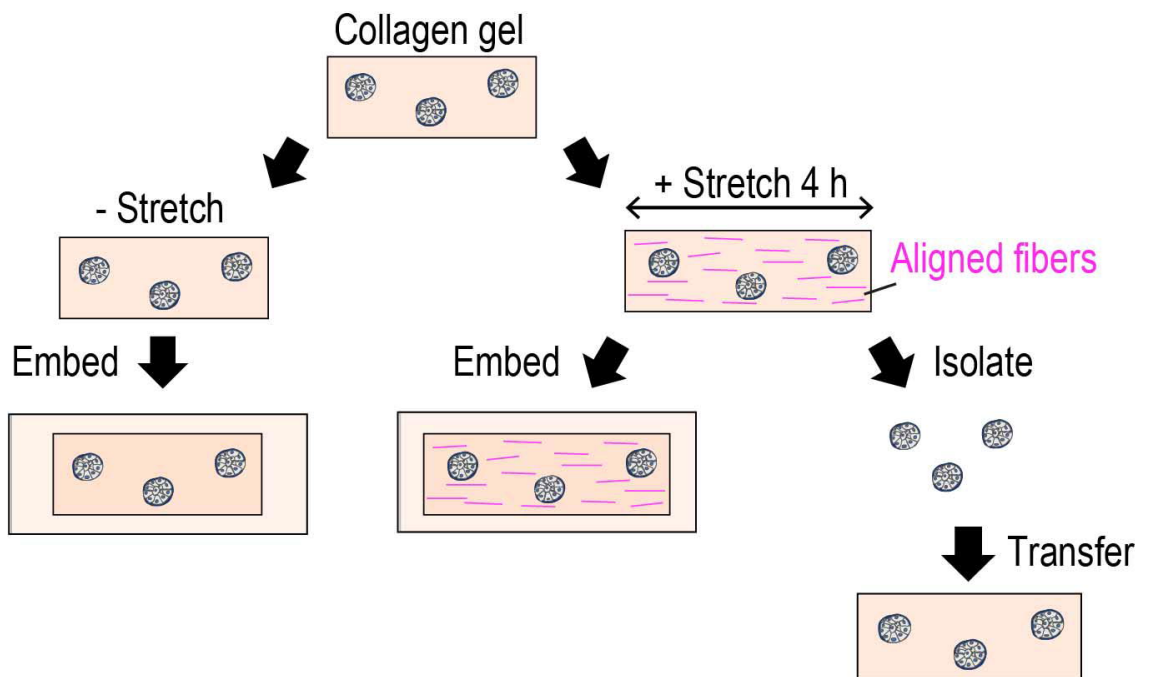

C

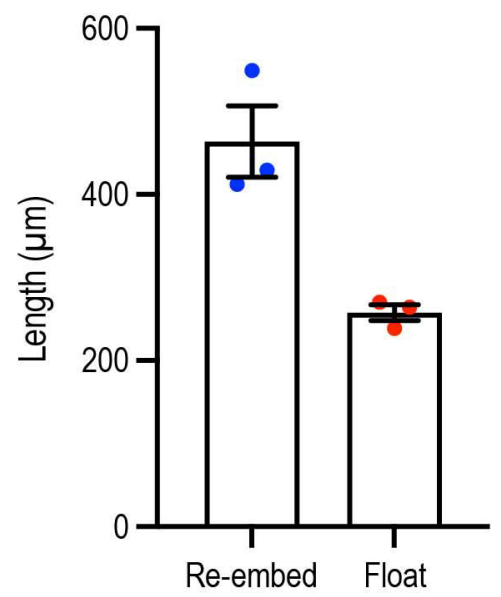

E
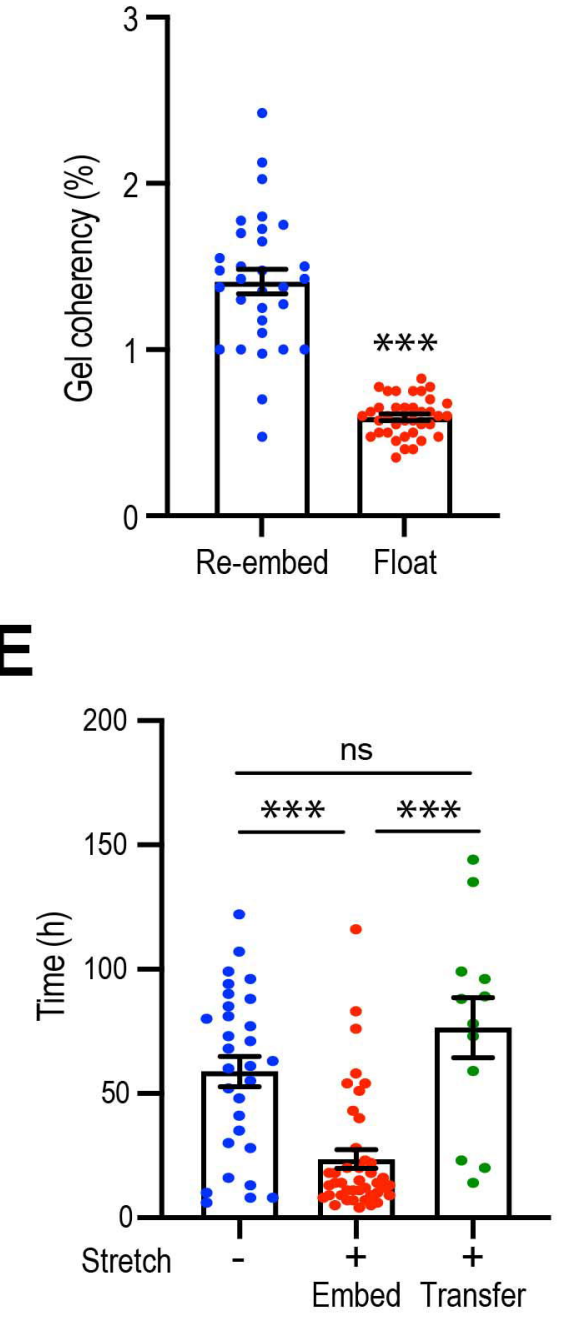

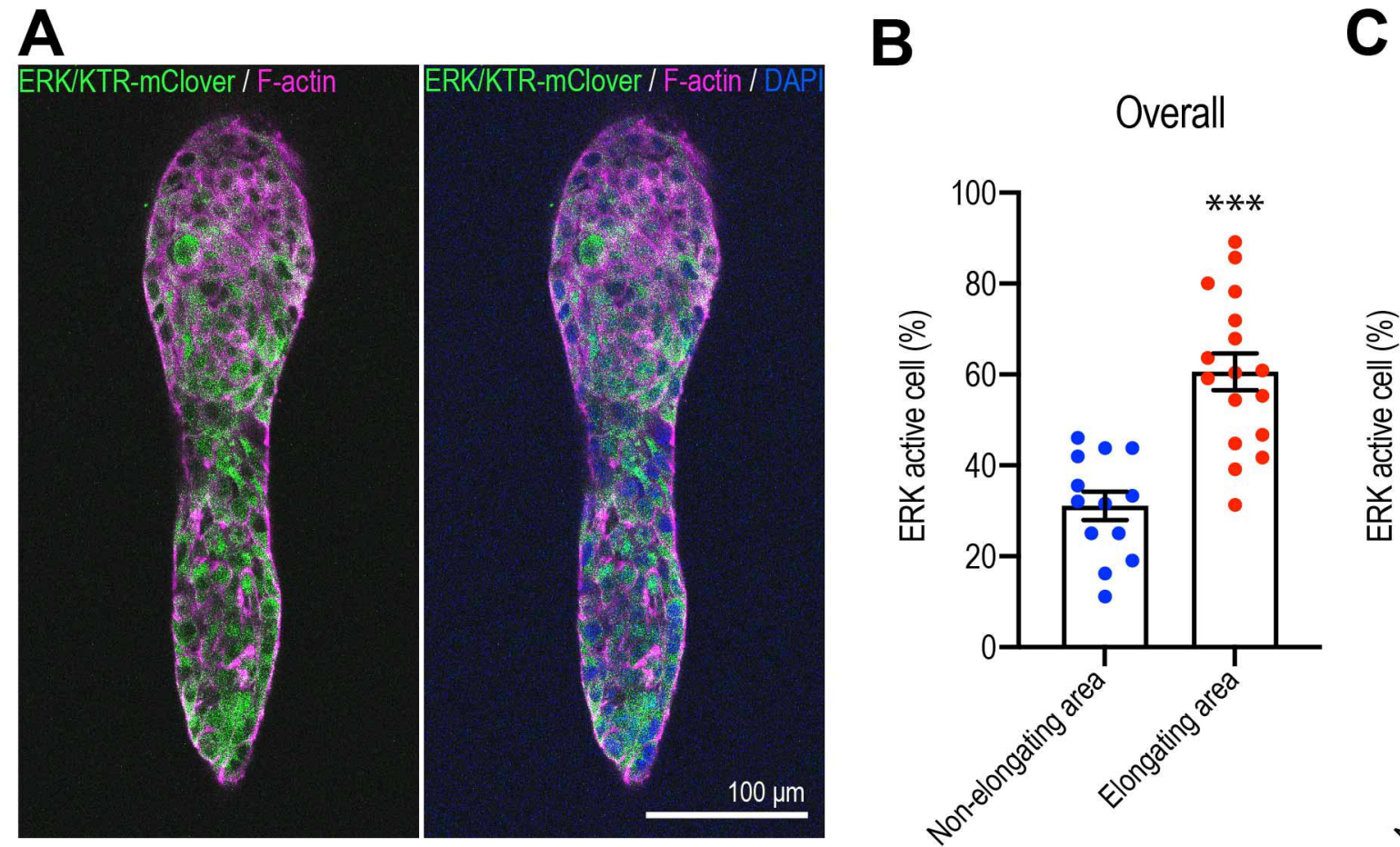

Overall

Surface
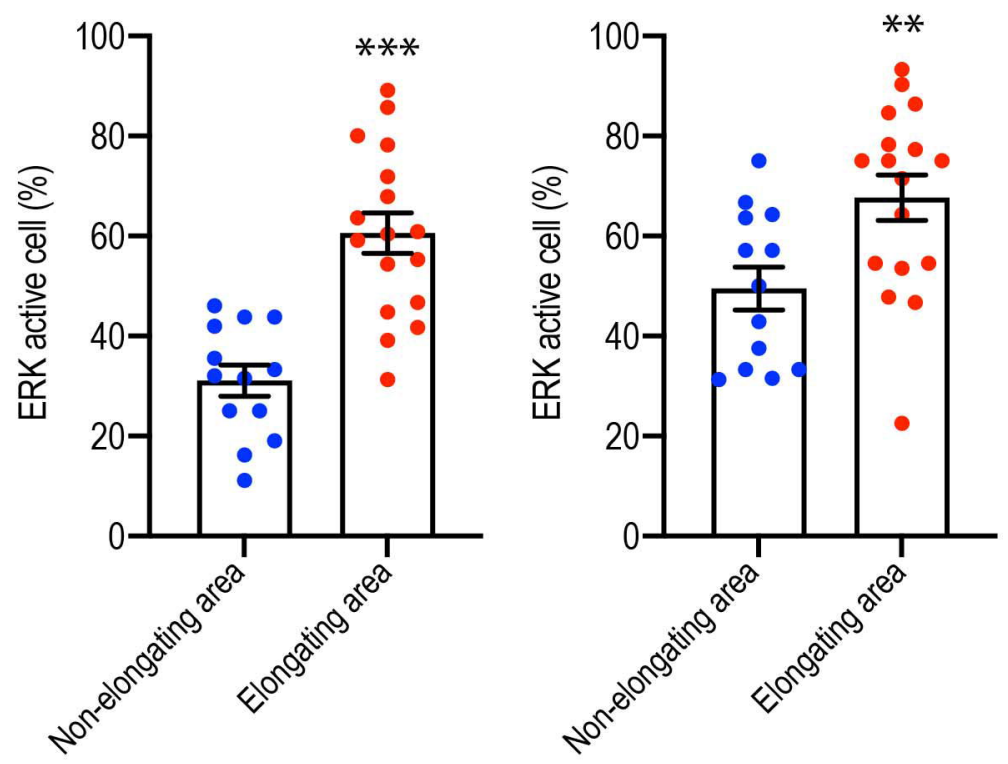

D

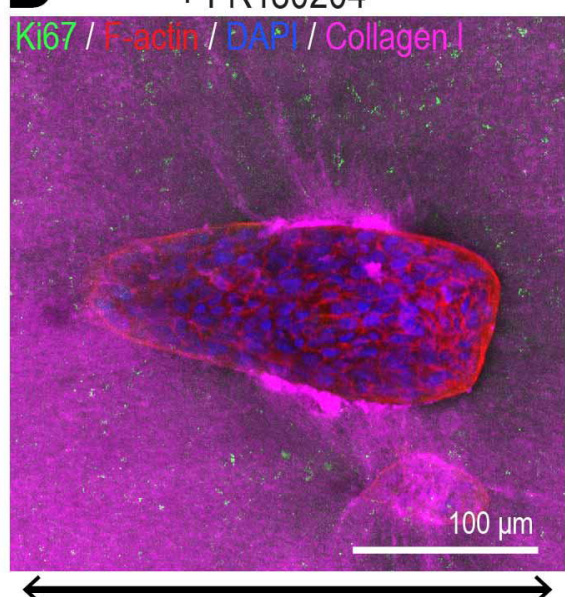

Stretching axis

F

$\frac{0}{2}$
100
$80-$
$60-$
$40-$
$20-$
0

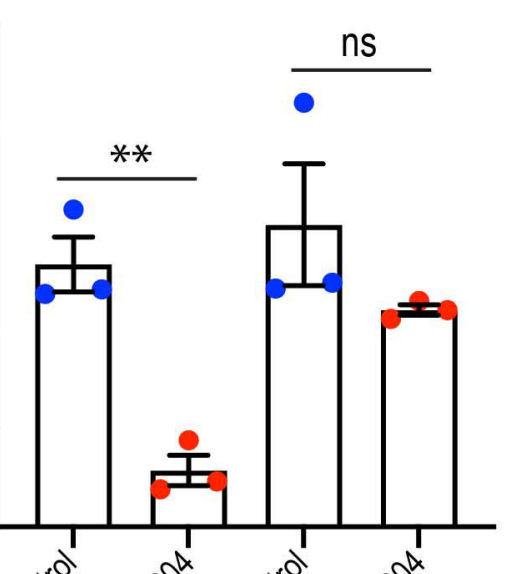

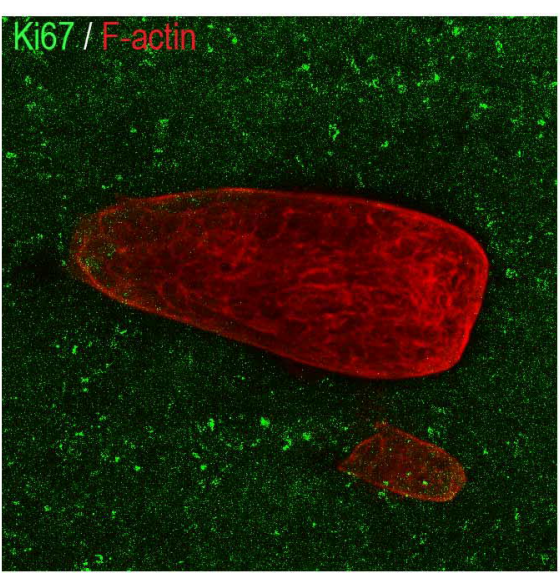

G

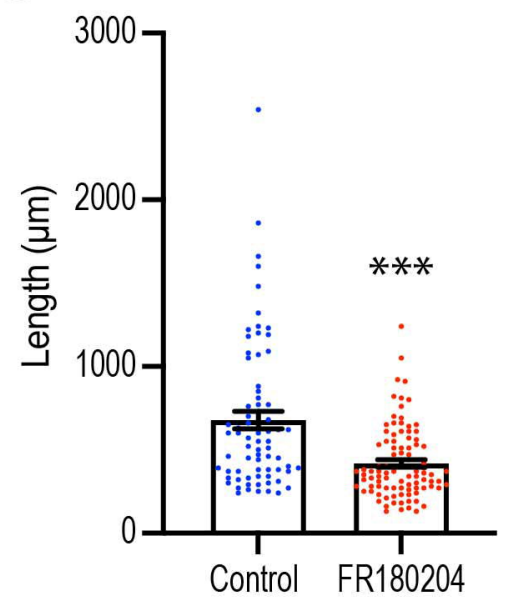

E

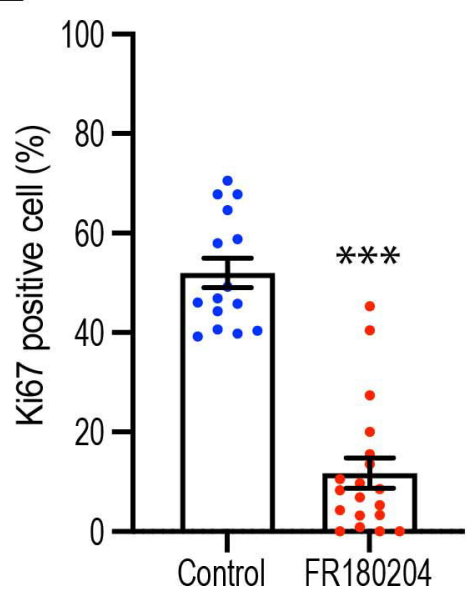

H

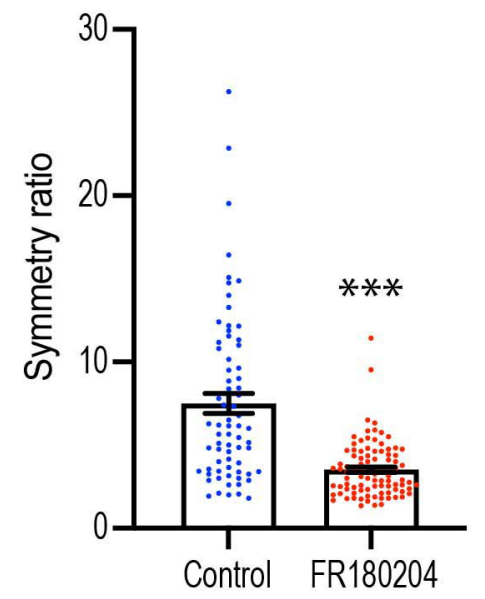


Katsuno-Kambe et al.

Figure 6-figure supplement 1

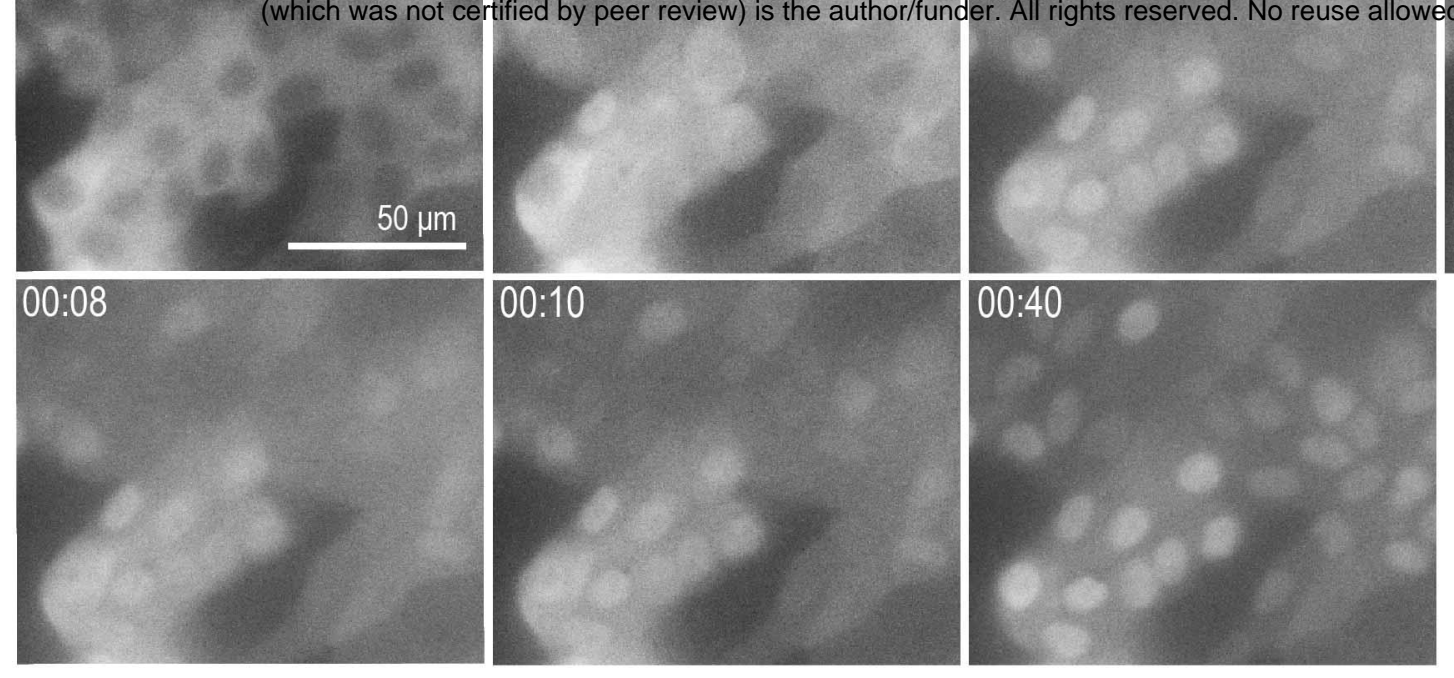

B

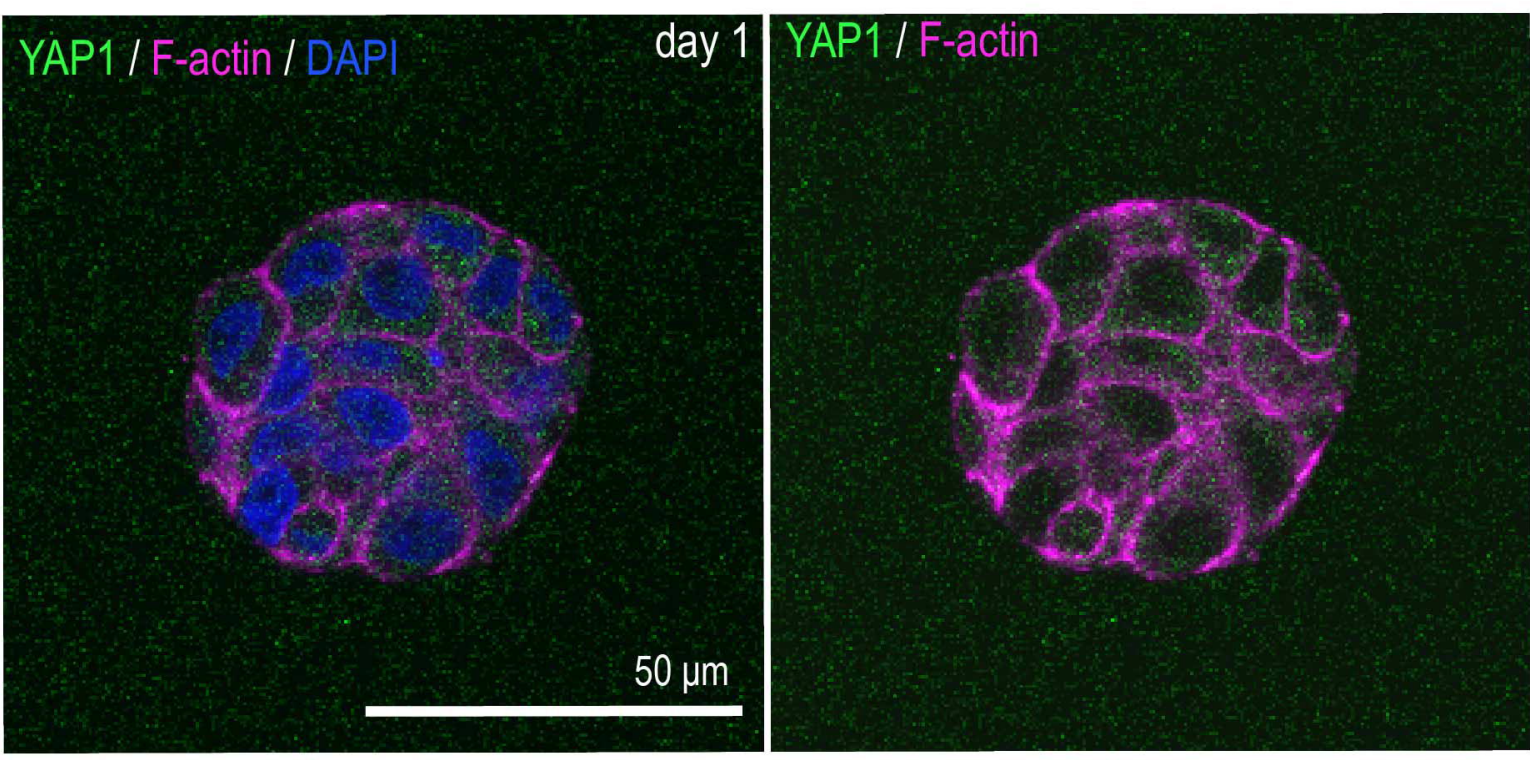

YAP1 / F-actin / DAPI

day 8

$100 \mu \mathrm{m}$

YAP1 / F-actin

A 00:00 + FR180204
ERK/KTR-mClover 0
bioRxiv preprint doi: https://dolic

\begin{tabular}{|c|}
\hline $00: 02$ \\
\hline
\end{tabular} \\ (which was not certified by peer review) is the author/funder. All rights reserved. No reuse allowed without permission \\ $\mathbf{A}_{00000}$ \\ holder for this preprint}

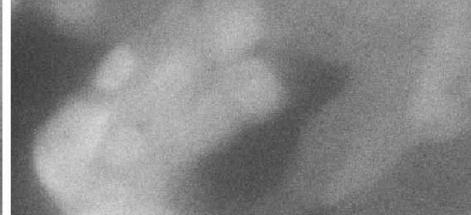

C

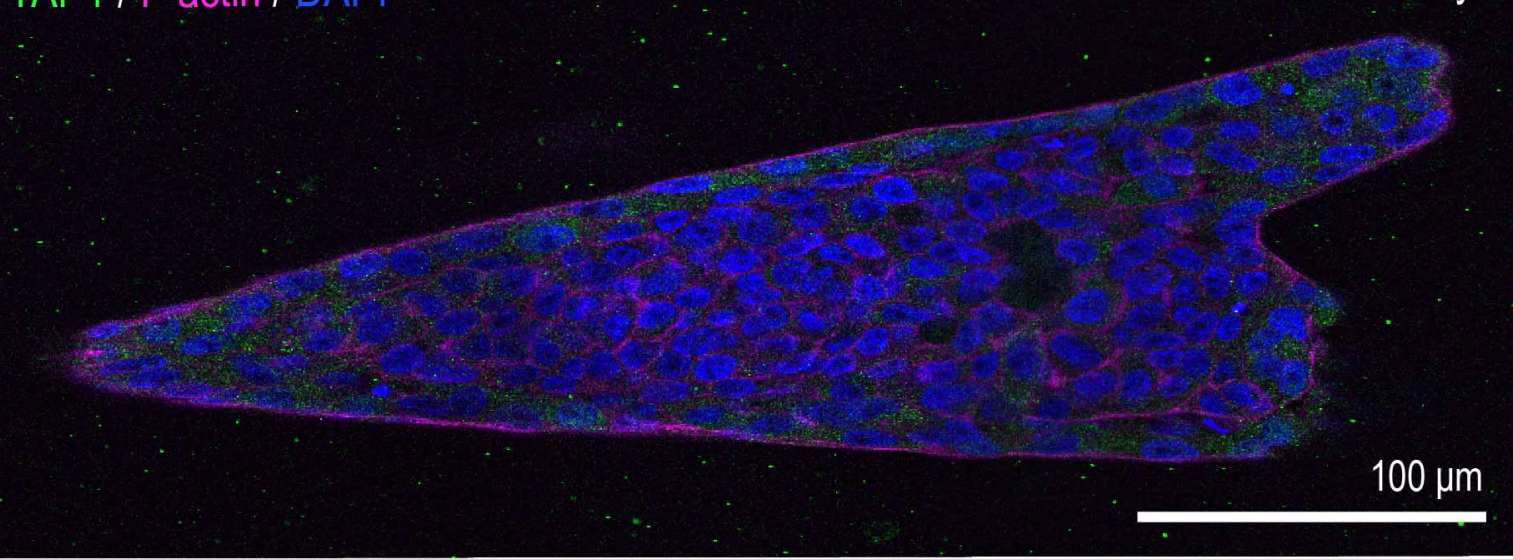


A

Collagen polarisatioin

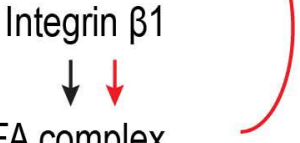

FA complex

Cell proliferation

D

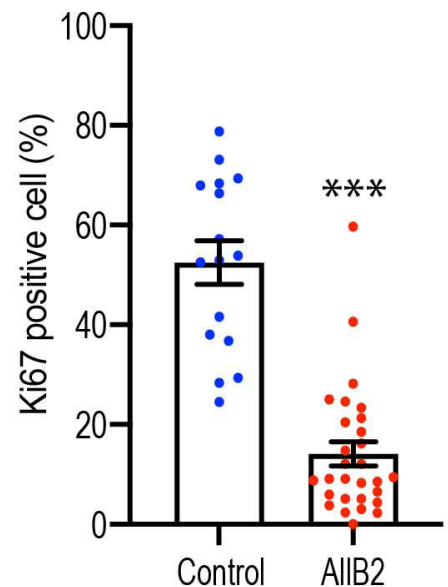

H

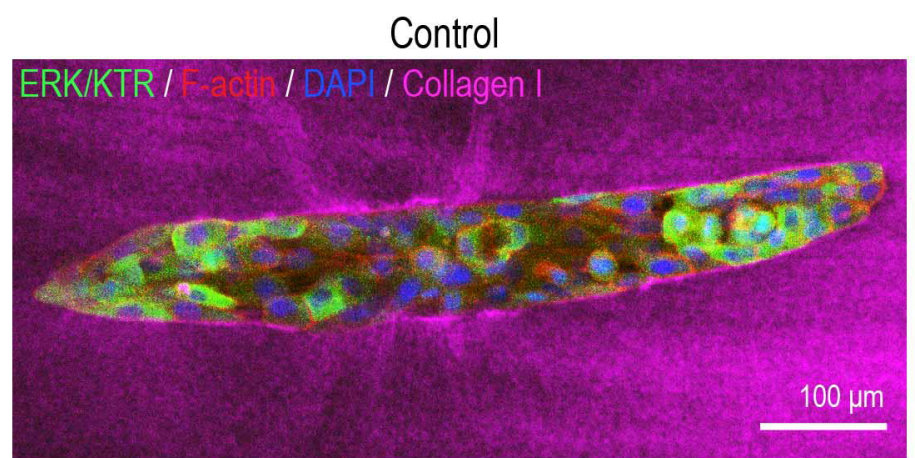

Stretching axis
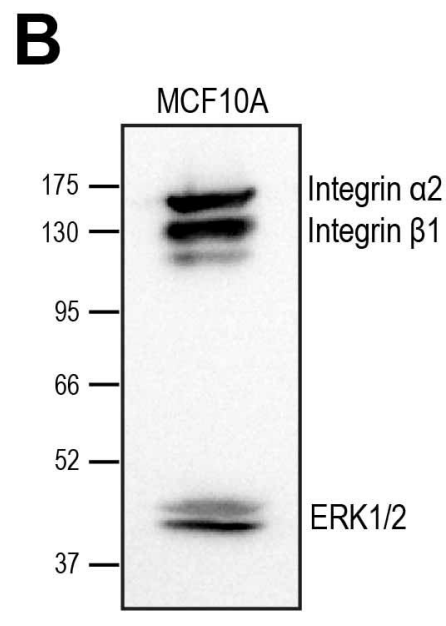

E

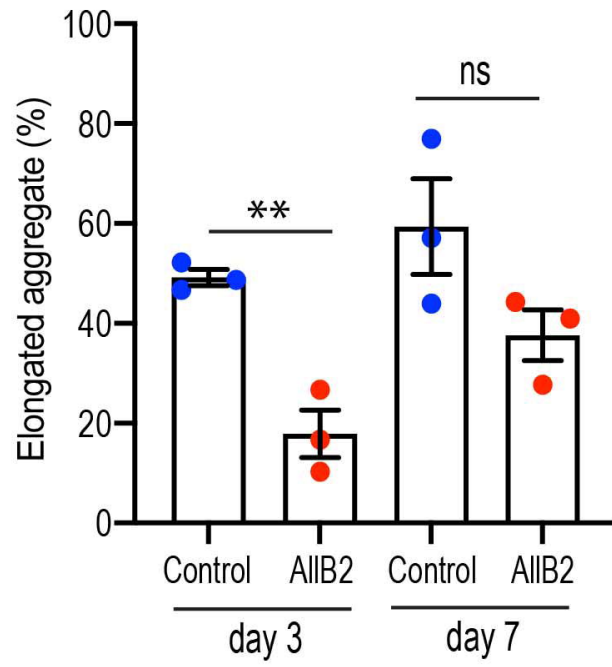

+ AllB2

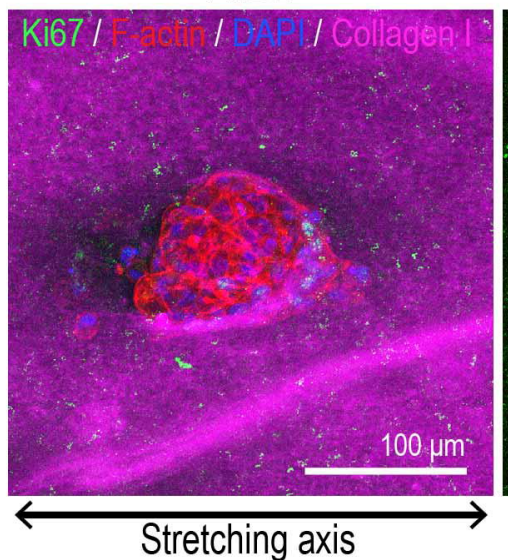

F

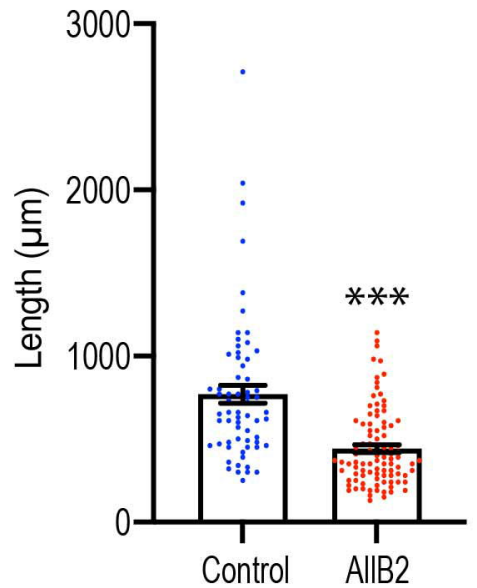

G

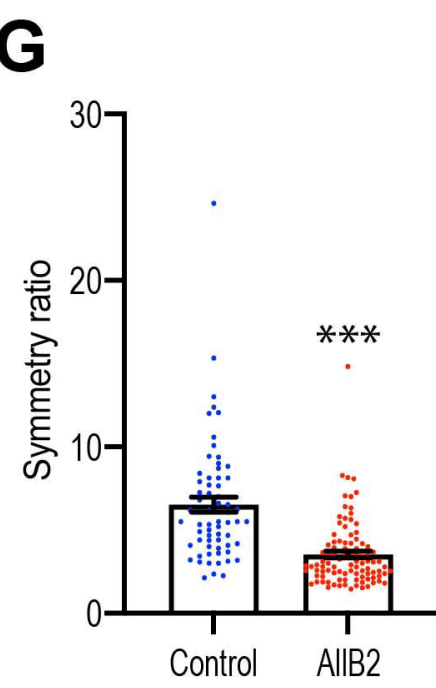

+ AllB2

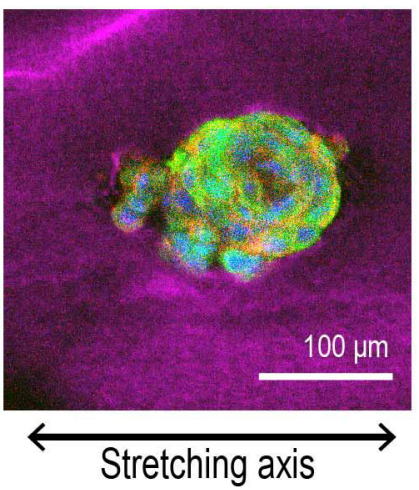

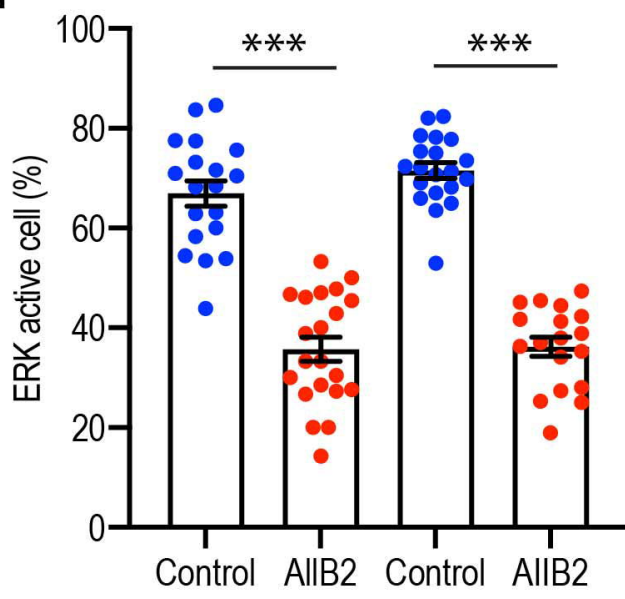

day 2

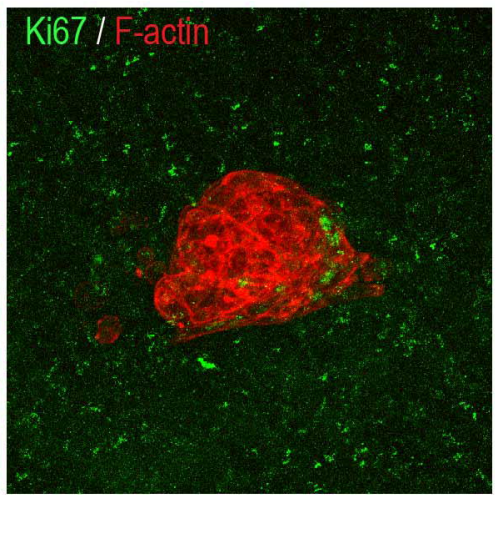

$$
\text { . }
$$


(i)
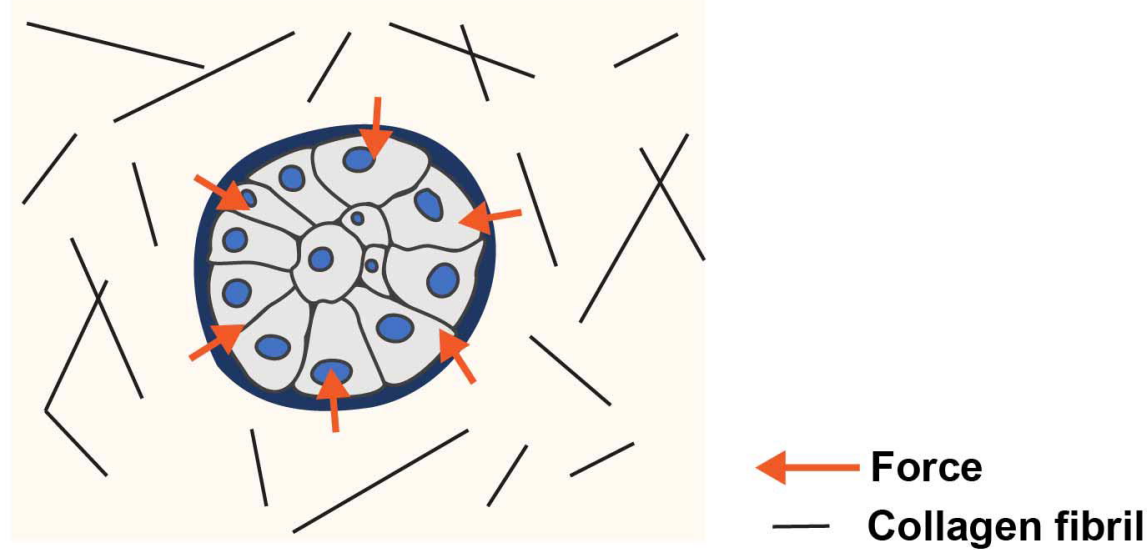

(ii)

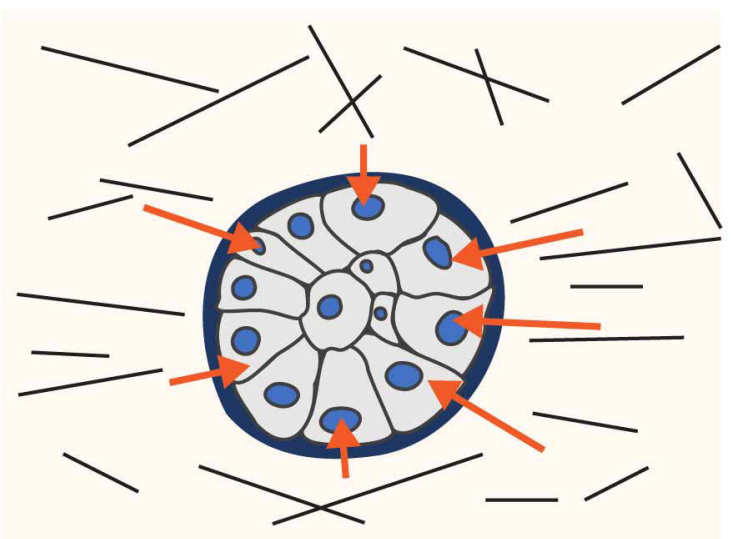

(iii)
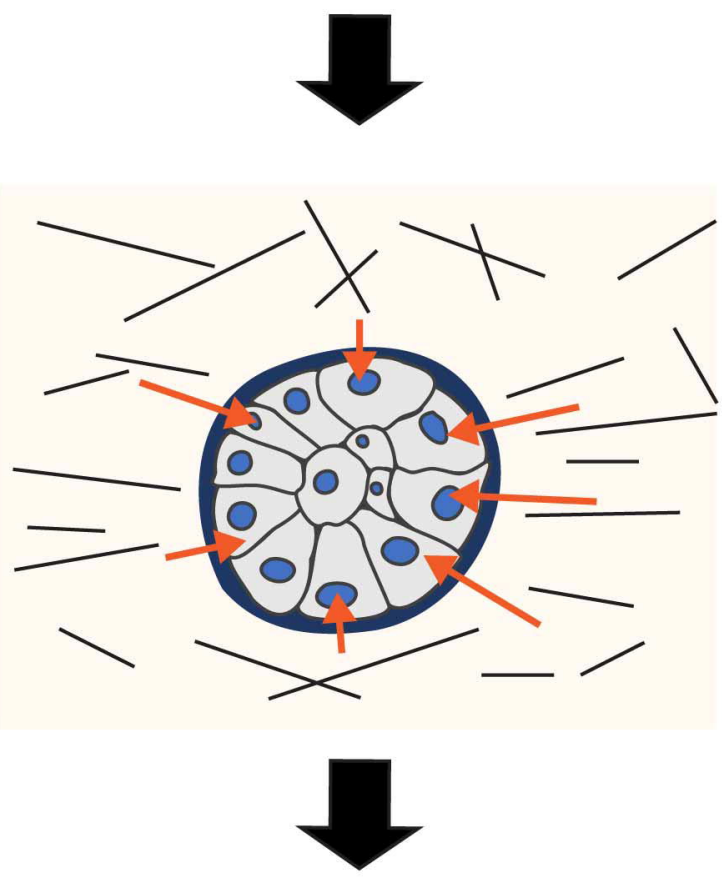

Collagen fibril

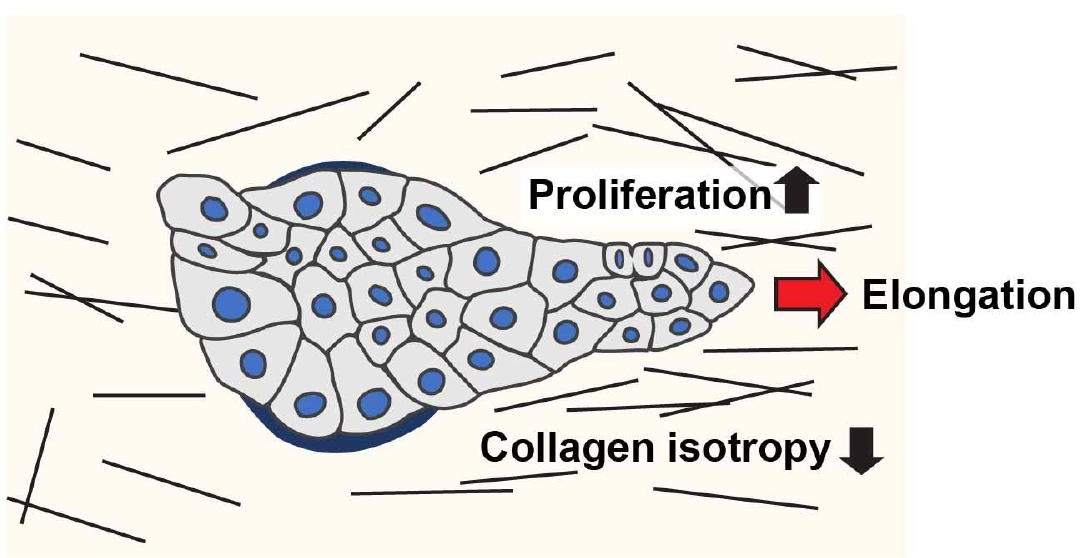

Katsuno-Kambe et al. 